\title{
1 The impassable gap between experiential and symbolic values
}

2 Basile Garcia (1), Maël Lebreton (2,3), Sacha Bourgeois-Gironde $(4,5)$ \& 3 Stefano Palminteri $(1,6, \S)$

4 (1) Laboratoire de Neurosciences Cognitives Computationnelles, Département d'Etudes Cognitives, ENS, 5 PSL, INSERM, Paris, France;

6 (2) Paris School of Economics, Paris, France

7 (3) Swiss Center for Affective Science, Faculty of Psychology and Educational Sciences, University of 8 Geneva

9 (4) Institut Jean Nicod, Département d'Etudes Cognitives, ENS, EHESS, PSL, CNRS, Paris, France

10 (5) Assas CRED - Université Panthéon-Assas - Paris 2, Paris, France

11 (6) Institute of Cognitive Neuroscience, Higher School of Economics, Moscow, Federation of Russia

$12 \S$ corresponding authors (basile.garcia@ens.fr, stefano.palminteri@ens.fr)

\section{Abstract}

14 To choose between options of different natures, standard decision models presume that 15 a single representational system ultimately indexes their subjective values on a common scale, regardless of how they are constructed. To challenge this assumption, we

17 systematically investigated hybrid decisions between experiential options, whose value is

18 built from past outcomes experience, and symbolic options which describe probabilistic outcomes. We show that participants' choices exhibited a pattern consistent with a

20 systematic neglect of the experiential values. This normatively irrational decision strategy

21 held after accounting for alternative explanations, and persisted when it bore an economic 22 cost. Overall, our results demonstrate that experiential and symbolic values are not 23 symmetrically considered in hybrid decisions, suggesting that they are not 24 commensurable and recruit different representational systems which may be assigned 25 different priority levels in the decision process. These findings challenge the dominant 26 models commonly used in value-based decision-making research. 


\section{Introduction}

28

Standard models of economic decision-making generally assume a two-step decision process, where individuals identify and assign values to available options, and ultimately pick the option with the highest subjective value (1-3). The values attributed to individual options can derive from different sources. On the one hand, a priori neutral stimuli acquire positive or negative experiential values after association with past outcomes (rewards and punishments) (4-6). On the other hand, the explicit description of an option's possible outcomes and their probabilities are combined to form a subjective expected value (710). Such explicit descriptions may take many different forms, including written language (from simple vignettes to fully specified numerical variables), a symbolic code communicating the decision variables (payoffs and probability) in an unambiguous manner, or a combination of the two (11).

In the standard two-step model, the way option values are built (via experience or description) is only peripheral to the decision process itself, meaning that experiential and symbolic values converge to a central valuation and decision-making system $(3,12-16)$. Thereby, choices between experiential and symbolic options should present no particular challenge, because their values are translated into an internal common currency, allowing an unbiased comparison between these differently generated option values. This normative point of view is indirectly supported by the fact that the neural correlates of experiential and symbolic values largely overlap in the so-called brain valuation system $(17-20)$.

However, several lines of evidence in behavioral decision-making research question the idea of a central valuation system. In fact, it is now a very well established that, when studied separately, experience-based and description-based choices display different properties: a phenomenon referred to as the description-experience gap (21-24). This difference in the subjective valuation of experiential and symbolic options poses a direct, theoretical challenge to the idea of a central valuation system (25). This rather suggests the existence of modality-specific valuation systems, relying on distinct cognitive 
57 representations, which would hinder, if not impede, the comparison between experiential 58 and symbolic options.

59 Strikingly, this key prediction has not been directly assessed, because studies usually 60 consider separate sets of decision problems for experiential and symbolic options (26, 61 23). Thereby, to date, very little experimental evidence has formally assessed the 62 commensurability of experiential and symbolic option values, nor their mapping into a 63 central or different valuation systems $(27,28)$. This is particularly problematic considering 64 that hybrid choices seem to be the norm rather than the exception in our modern societies 65 where descriptive information is omnipresent. For example, everyday situations like 66 choosing between our favorite restaurant (experience) and a new one with good review 67 (description) is a prototypical example of such a hybrid decision.

68 To fill this gap and challenge the commensurability of experiential and symbolic values, 69 we designed a new behavioral protocol. The experiment started with a learning phase 70 during which human participants repeatedly faced abstract cues paired with probabilistic 71 outcomes, thereby learned to associate experiential expected-values to the originally 72 neutral symbols. After this phase, participants were asked to make hybrid choices 73 between the experienced symbols and described lotteries visualized as colored pie-charts 74 (a standard way to represent value symbolically) (11). When making hybrid choices, 75 participants treated the two kinds of options asymmetrically and, specifically, were 76 neglecting experiential values. This asymmetry was robust across seven experiments, 77 where we controlled for many possible alternative explanations, such as, insufficient 78 learning, generalization issues or lack of incentives. Overall, the relative neglect of an 79 option's value conditional on its source is consistent with the idea that different types of 80 values - such as experiential and symbolic - may involve different representational 81 systems, resulting in their incommensurability. 
A

Learning (LE) - 120 trials

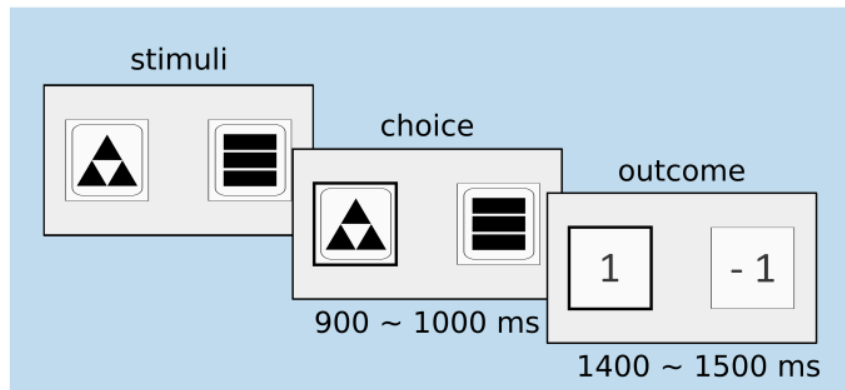

\section{Experiential-Symbolic (ES) - 88 trials}

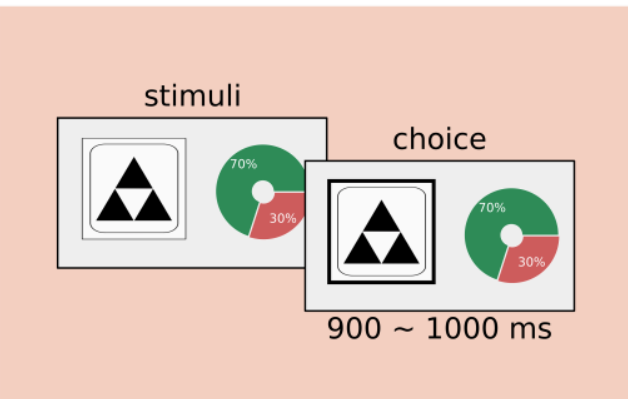

B

Hypotheses
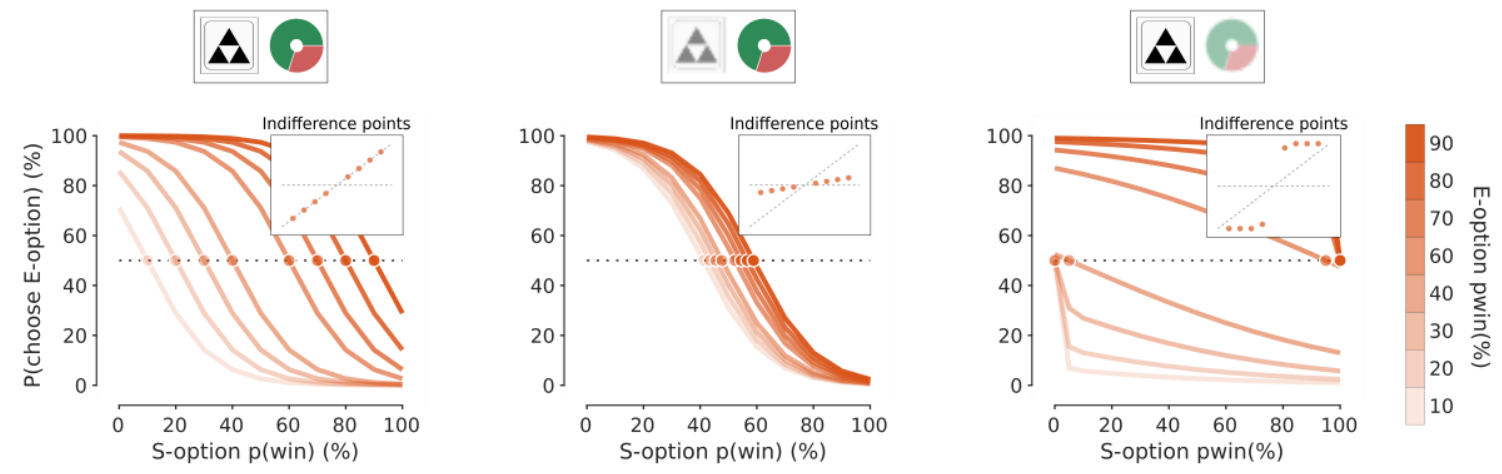

\section{Options}
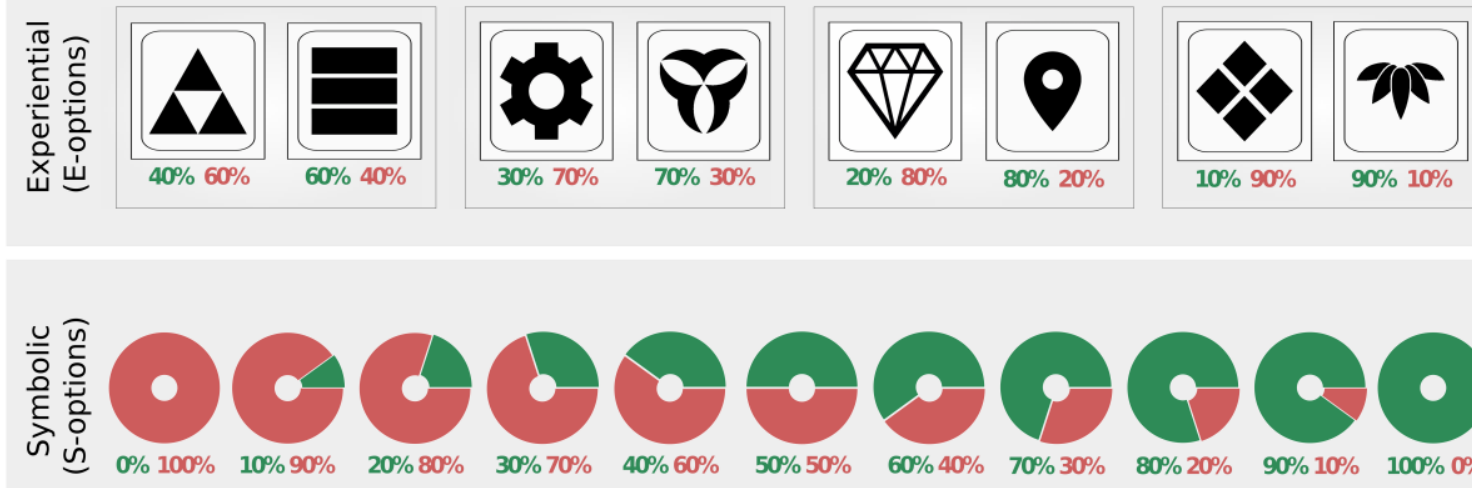

D

Experiment structure

\section{Learning phase}

Learning (LE)

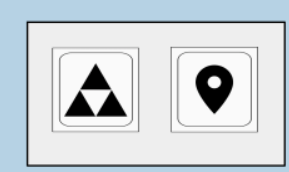

120 trials
Post-learning phase (w/o feedback)

Experiential-Symbolic (ES)

Experiential-Experiential (EE)

Stated Probability (SP)

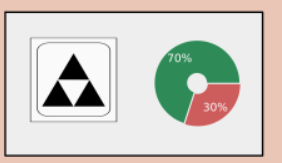

88 trials

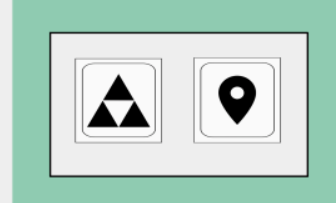

56 trials

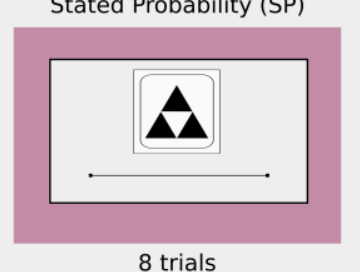

8 trials 
Fig 1. Behavioral tasks, hypotheses, option values and experimental protocol. (A) The leftmost panel displays successive screens of a typical trials in the learning phase (LE). The LE-phase consists in a twoarmed bandit task with fixed (4 or 2 - in Exp. 4) pairs of abstract cues (E-options) and contained 120 trials. The rightmost panel displays successive screens of a typical trials in the Experiential-Symbolic choice phase (ES). The ES-phase consists in binary choices between a lottery (standardly materialized as a piechart) and a symbol previously presented in LE-phase. In most experiments, the EE phase lasted 88 trials (8 E-options $\times 11 \mathrm{~S}$-options). Durations are given in milliseconds. (B) The panels illustrate three possible hypotheses on how participants could make choices in the ES-phase. In each panel the probability of chosen the E-option is plotted against the value of the S-option (expressed as probability of winning a point). The insets represent the indifference points (where the curves cross $50 \%$; of not unbiased indifference points should lay on the diagonal). The color of the curves indicates the value of the E-option (lowest: light orange; highest: dark orange). The leftmost panel illustrate the default hypotheses according to which E-options and S-options are fully commensurable and therefore the curves cross $50 \%$ (indifference point) at exactly the value of the E-option. The central panel illustrates experiential value neglect scenario according to which ES-choices are determined (almost) uniquely by the value of the S-options. Finally, the rightmost panel illustrates the symbolic value neglect scenario, accordingly to which ES-choices are determined (almost) uniquely the value of the E-options. (C) The panel displays the options values. The topmost part shows how E-option were organized in learning contexts (in all experiment except Exp. 4 and 7; of note, the attribution of the value to the symbols was randomized across participants). The bottommost part shows the lotteries used in the ES phase (in all experiment except Exp. 7). (D) The experiments were structured as follows: they all started with a learning phase (LE), where participants made choices between abstract symbols and received feedback information. After the LE phase, participants were asked to make repeated choices between each E-option and several lotteries (see Fig. 1A and Fig. 1C). From Experiment 5 on, participants were also asked to make choice between E-options that were not necessarily presented together. Finally, we assessed the stated probability (SP) of winning for each symbol by asking participants to explicitly rate each E-option, following a probability matching procedure (29).

\section{Results}

111 We conducted a series of experiments structured in two main phases, one allowing the 112 formation of subjective values from the experience of past outcomes, and a second where 113 these experiential options (E-options) were presented against options whose subjective 114 values were described by symbolic means (S-options) (Fig. 1A). During the first (or 115 learning: LE) phase, E-options were materialized by abstract shapes that provided no 116 explicit information concerning the expected value (EV) of the option. During the LE 117 choices, E-option values could therefore only be inferred from the history of gains $(+1$ 118 point) and losses (-1 point) associated to a specific cue. E-options were presented in four 119 fixed pairs, each featuring an EV-maximizing and an EV-minimizing option. 120 Subsequently, in the Experiential-Symbolic (ES) phase, participants were asked to make 121 choices between the very same E-options of the previous phase and pie-charts explicitly 
123 the main focus of this paper, we thereafter delineate three plausible hypotheses 124 concerning the behavioral output of this phase.

125 First, assuming that the subjective values of the E- and S-options are mapped into a common scale (common currency hypothesis), participants should make unbiased 127 decisions in the ES phase. Accordingly, the probability of choosing, say, the E-option, will be jointly determined by the EV of the E- and the S-option (Fig. 1B: left). In other terms, for a given E-option the inferred indifference point will precisely correspond to S-options with equal EV.

131 Alternatively, the possibility that subjective values are constructed and represented in a modality-specific way (representational gap hypothesis) entails that E- and S-options are not readily commensurable. This situation could lead to two possible scenarios. In one 134 of them, participants make random choices in the ES-phase. In the other scenario participants could prioritize one of the two sources of information. Within this scenario, participants could resolve the tension between E- and S-options basing their choices 137 primarily on the explicit symbolic values provided by the lotteries. In other terms, participants would pick the lottery, when positive, and reject it when negative, as if the Eoption values were neglected and regressed to zero (experiential value neglect; Fig. 1B: $\mathrm{mid})$. In the other case, participants would present an over-reliance on experiential values and would display the opposite pattern: accept or reject an E-option without considering 142 the S-option value (symbolic value neglect; Fig. 1B: right). Crucially, the ES phase of our 143 experiments allows to tease apart these different scenarios by analyzing the probability 144 of choosing an E-option as a function of the S-option being presented. More precisely, 145 taking each E-option separately and uncovering the S-option (value) at which a 146 preference shifts from the former to the latter provides us with an estimate of how much 147 a participant values an E-option. Quantifying the relation between E-options and S148 options boils down to inferring indifference points (i.e., when the probability of choosing 
150 (Fig. 1B: insets).

A
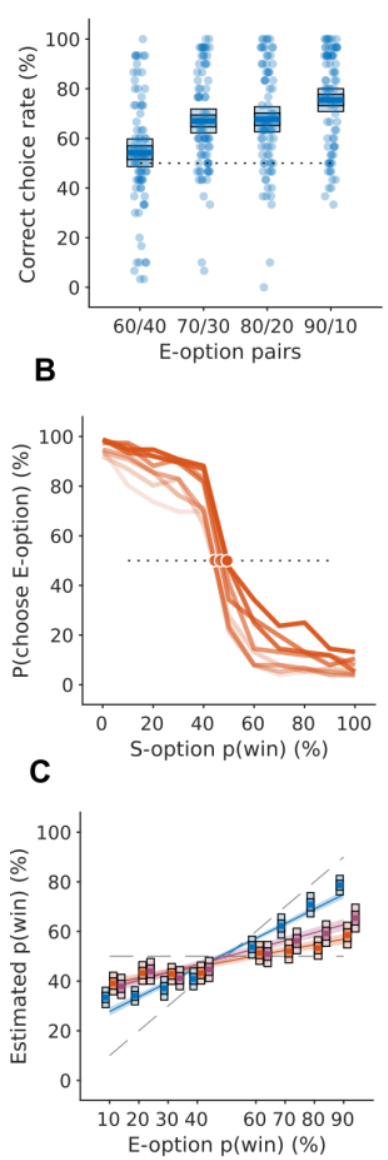

D

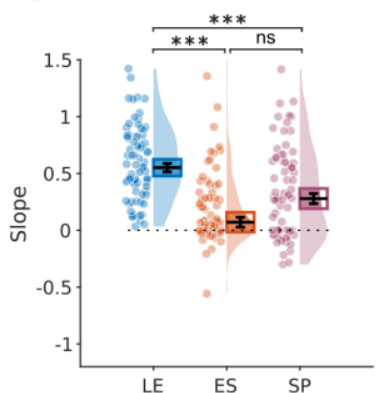

Exp. 2
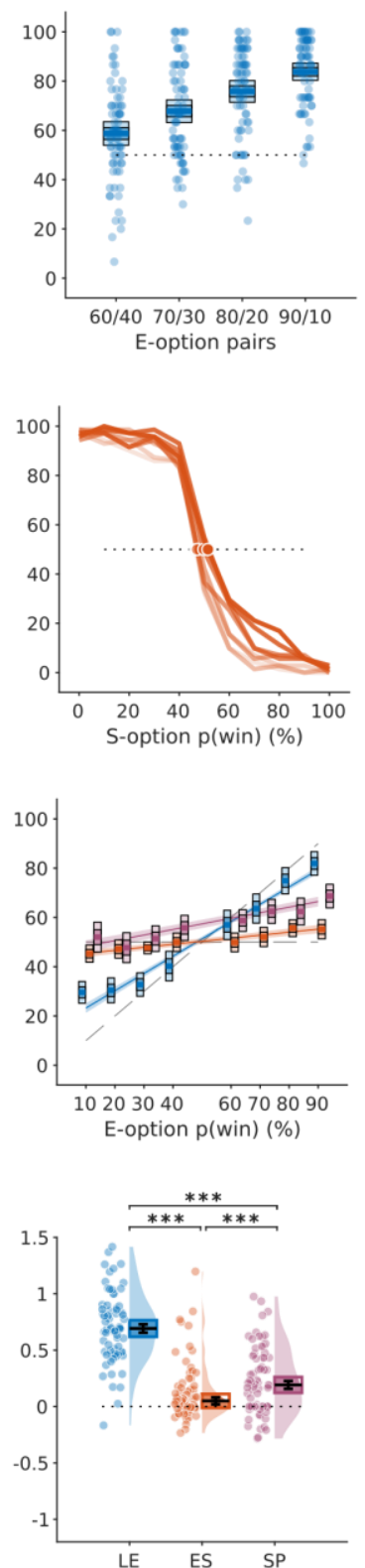

Exp. 3
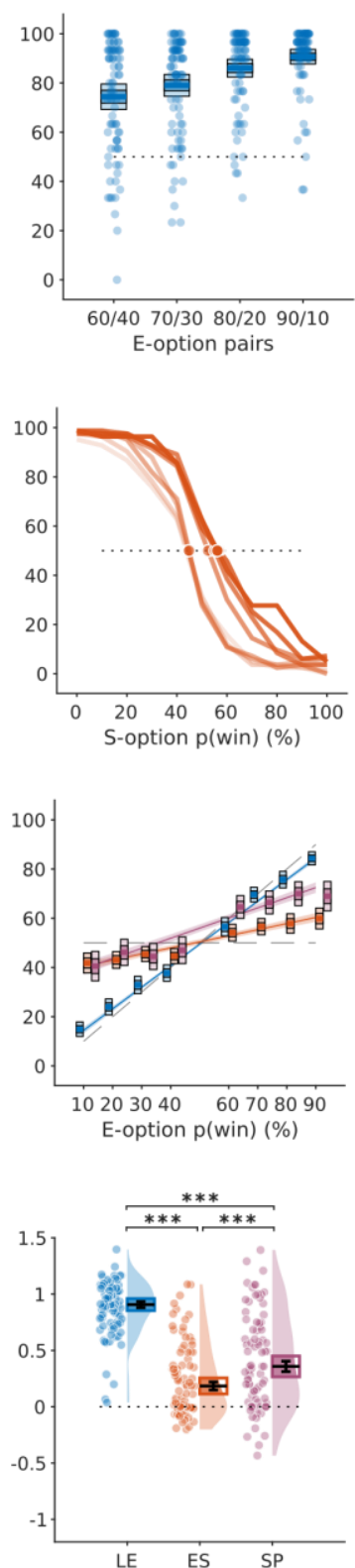

Exp. 4
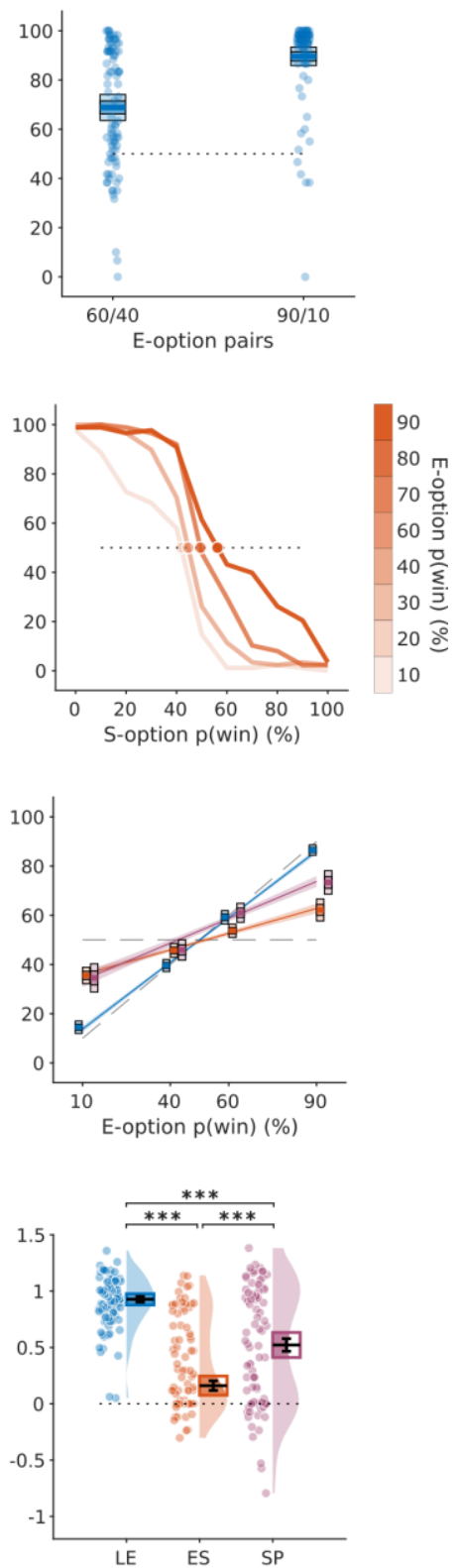

Fig 2. Raw behavioral results and inferred option values in Experiments 1-to-4. (A) Correct choice rate grouped per learning context in the LE phase, where '40/60' designated the hardest decision problem, '10/90' the easiest decision problem. The dark blue line indicates the mean, the mid-dark blue indicates the standard mean error, and the light blue indicates a 95\% confidence interval. The dotted line indicates chance (or random) responding (50\%). (B) Average probability of choosing an E-option over a S-option during ES phase. The color of the curves indicates the value of the E-option (lowest: light orange; highest: 
dark orange). Dots represent the empirical indifference points, the value of a lottery that corresponds to a probability of choosing the symbol $50 \%$ of the times. (C) The panels represent for each symbol the inferred value (as expressed by the probability of winning; $p($ win)) as a function of the actual value. ES estimates are represented in orange, LE estimates in blue and SP estimates in pink. In the data-boxes, the dark tone line represents the mean, mid-dark tone the standard mean error, light tone a $95 \%$ confidence interval. The lines represent linear regression (dark tone), and the average standard mean error (light tone). (D) Comparison of individual inferred slopes obtained from linear fit (see Fig. 2C) in the three modalities (LE, ES and SP in blue, orange and pink, respectively). The black lines represent mean and standard error of the mean. The colored boxes represent $95 \%$ confidence interval. The shaded area probability represents density functions. ${ }^{* *} p<0.001$ paired sample t-tests.

\section{First evidence for the experiential value neglect scenario}

In the LE phase of the first experiment $(\mathrm{N}=76)$, we presented pairs of $\mathrm{E}$-options in an interleaved manner (i.e., E-option pairs are distributed randomly in the sequence of trials) and we displayed only the outcome of the chosen option (partial feedback) (Fig. 2A, Exp. 1). Apart from the most difficult learning context (60/40), choice accuracy was above chance level for all E-option pairs $(\mathrm{T}(75)=1.5, \mathrm{P}>.05 ; \mathrm{T}(75)=10.98, \mathrm{P}<0.001)$, thus indicating that participants aimed at (and managed to) maximize expected value. Furthermore, accuracy was modulated by the difference in expected value (i.e., the decision value) of the E-option pair. Choice accuracy increased as a function of the decision value $(\beta=0.077, T(300)=2.16, P<0.05 ; \beta=0.08, T(300)=2.35, P<0.05 ; \beta=0.21$, $\mathrm{T}(300)=5.94, \mathrm{P}<0.001$ ), thus indicating that participants' behavior was sensitive to the specific $\mathrm{EV}$ of $\mathrm{E}$-options involved in a given pair.

Regarding analysis of the ES phase, the probability of choosing an E-option in an ES decision was largely determined by the S-option EV-value and the preference shift abruptly occurred around S-option EV equal to zero (i.e., $P(+1)=P(-1)=0.5$ ). Despite clear proofs of successful value learning and encoding during the LE phase, ES phasechoice pattern was clearly consistent with the experiential value neglect scenario. (Fig. 2B: left).

To quantify and statistically compare the differences in preferences observed in the LE and the ES phase, we first estimated the theoretical subjective value of each E-option separately for the two choice types, proxied by its probability of winning a point: $p$ (win) (remind that the outcomes are fixed, so the expected value of different options only depend on their probabilities to win). Concerning the LE phase, we leveraged on a 
193 classical associative learning approach, where we assumed $p$ (win) to be iteratively 194 updated as a function of a prediction error-minimizing learning rule $(30,31,6)$. We were able to infer $\mathrm{p}$ (win) attributed to each E-option at the end of the learning process by fitting 196 this, rather parsimonious and standard, model.

197 Concerning the ES phase, subjective $p$ (win) estimates were inferred using the following 198 method: the probability of choosing a specific E-option over a S-option of various 199 expected values was assumed to take the form of a logistic sigmoid function. We fitted 200 those logistic functions to each E-option and individual, and used them to extrapolate the 201 indifference points indexing E-options' subjective $p$ (win).

202 Finally, to compare the overall valuation of the E-options in the LE and the ES phases, 203 we computed a measure of how well the subjective $p$ (win) estimates from each phase 204 matched the objective underlying probabilities, using slopes estimates from linear 205 regressions.

206 At this aggregate level, a slope equal to 1 corresponds to an unbiased representation of 207 E-options' $p$ (win), whereas a slope equal to 0 corresponds to random representations. In 208 our data, the slopes estimated from the LE phase were significantly higher and closer to 2091 compared to those estimated from ES-choices (T(75)=6.53, $\mathrm{P}<.001)$ (Fig. 2C: left). 210 Thus, ES decision problems feature a specific neglect of E-option values, as if hybrid 211 choices prioritized the value of the symbolic options over an unbiased comparison of 212 experiential and symbolic values, thereby confirming the experiential neglect hypothesis.

213 We ruled out a first trivial interpretation for this result, by only including in the analyses 214 participants that performed at $100 \%$ of correct response in catch trials (i.e. trials involving 215 choices between two S-options; see Supplementary Materials), disseminated across 216 the ES phase to ensure the participants' capacity to understand the symbolic 217 representation of the probabilities.

218 In the following sections of the paper, we provide additional evidence in favor of the 219 experiential neglect hypothesis by progressively ruling out alternative interpretations via 220 additional measures and experiments. 


\section{Ruling out insufficient learning and forgetting}

223 While the experiential neglect pattern observed in the ES phase is consistent with the 224 idea that E-options and S-options are not equally considered in the decision process, it is 225 also consistent with a much more trivial hypothesis: insufficient learning. Despite 226 reinforcement learning model fitting suggesting otherwise (see Fig. 2C: left), it is indeed 227 possible that the neglect of E-option in the decision is caused by an imperfect and noisy 228 E-option value representations at the end of the learning phase. To rule out this alternative 229 interpretation, we devised a series of experiments where we changed the LE phase in order to improve learning, while keeping the (average) option values the same. In a second experiment (Exp. 2; $\mathrm{N}=71$ ), we therefore presented decision problems as blocks (rather than interleaved as in Exp. 1), so as to improve performance and option identification by preventing the saturation of working memory (32). In a third experiment (Exp. 3; $\mathrm{N}=83$ ), we additionally provided the outcome information concerning the unchosen option - a manipulation known for increasing accuracy $(33,34)$. Finally, on top of these variations, in a fourth experiment (Exp. $4 ; \mathrm{N}=88$ ) we also reduced the number of decision problems of the LE phase to two, such that each decision problem was presented for twice as many trials as in experiments $1-3$, thereby reducing the uncertainty about the options' outcomes. These manipulations were successful in significantly increasing decision accuracy in the LE phase (Exp. 1: $0.66 \pm 0.01$; Exp. 2: $0.71 \pm 0.01, \beta=0.05$, $\mathrm{T}(314)=2.28, \mathrm{P}<0.05$; Exp. 3: $0.82 \pm 0.01, \beta=0.16, \mathrm{~T}(314)=7.17, \mathrm{P}<0.001$; Exp. 4: $0.79 \pm 0.01 ; \beta=0.13, T(314)=5.8, P<0.001)$, while avoiding ceiling performance issues.

243 Indeed, even in the easiest experiments, accuracy was still significantly modulated by the 244 decision values; for instance, the accuracy in the more difficult decision problem (60/40) 245 was always lower compared to the easiest one ('90/10') ( $T=5.81, P<0.001 ; T=8.81$, $246 \quad P<0.001)$.

247 Crucially, the remarkable increase in the LE phase accuracy of the new experiments $248(107 \%-124 \%$ of Exp. 1) was not paralleled by detectable qualitative differences in ES 249 phase choice patterns (Fig 2B). In other terms, the experiential value neglect persists 250 despite the uncertainty concerning the E-options' values being considerably reduced (via 
251 blocked design, complete feedback and increasing the number of trials per decision 252 problem).

253 To quantitatively characterize this claim, we estimated the subjective $p($ win) for each $E$ 254 option separately for the LE and the ES phases and fitted a linear regression between the estimated subjective $p$ (win) and their true values (as described above). Confirming the efficiency of our manipulations in increasing learning performance, the LE-inferred slopes increased significantly across experiments $(\operatorname{Exp} .2: \beta=0.11, T(942)=5.98, P=0.055$; Exp. 3: $\beta=0.28, T(942)=6.5, P<0.001$; ; Exp. 4: $\beta=0.31, T(942)=7.27, P<0.001)$. Critically, the ES slopes were not modulated across experiments aside from Exp. 4 (Exp. 2: $\beta=-$ 0.1, $T(942)=-1.76, P=0.07$; Exp. 3: $\beta=0.02, T(942)=6.5, P=0.67$; Exp. 4: $\beta=0.11$, $\mathrm{T}(942)=2.06, \mathrm{P}<0.05$ ) (Fig. 2D). Overall, LE-inferred slopes were significantly higher than the ES slopes in all experiments (Exp. 2: $\mathrm{T}(70)=11.74, \mathrm{P}<0.001$; Exp. 3: $\mathrm{T}(82)=15.8, \mathrm{P}<0.001$; Exp. 4: $\mathrm{T}(87)=11.64, \mathrm{P}<0.001$; Fig. $2 \mathrm{E})$, and the asymmetric effects of the manipulations on the LE versus ES phases translated into a significant interaction between the choice modality (ES and LE) and the experiment number (Exp. 2: $\beta=-0.21, T(942)=-2.58, P<0.05$; Exp. 3: $\beta=-0.26, T(942)=-3.29, P<0.01$; ; Exp. 4: $\beta=0.2, T(942)=2.57, P<0.05)$.

The comparison between the first four experiments suggests that experiential value neglect is not a mere effect of insufficient learning. We indeed observe that an improved performance in the learning phase does not translate into a similar decrease of the experiential value neglect effect. However, independently of the quality of learning, it is also theoretically possible that participants forgot the E-option values when entering the ES hybrid choice phase, although the fact that the ES phase directly succeeded the LE phases within a matter of seconds makes it improbable. To rule out this possibility, in

275 Exp. 1-4, we asked participants to evaluate the E-options' $p$ (win) just after the ES phase, 276 by implementing a fully incentivized stated probability (SP) procedure (35). More 277 precisely, participants were explicitly asked to rate the probability of winning a point they 278 attribute to an E-option, by means of a numerical rating scale (Fig. 1D).

279 We then evaluated the quality of the E-option memory retention by regression these 280 stated probabilities against their true values. Note that because this elicitation happens 
281

282

283

284

285

286

287

288

289

290

291

292

293

294

295

296

297

298

299

300

301

302

303

304

305

306

307

308

309

310

311

after the ES phase, this SP-inferred slopes constitutes a lower bound of how well E-option values are learned and could be recovered during the ES phase. Yet, the SP-inferred slopes were systematically higher than the ES-inferred slopes and significantly so in Exp. 2, 3, 4 (Exp. 1: $\mathrm{T}(75)=2.62$, $\mathrm{P}>0.05$; Exp. 2: $\mathrm{T}(70)=3.42, \mathrm{P}<0.05$; Exp. 3: $\mathrm{T}(82)=4.38$, $\mathrm{P}<0.001$, Exp. 4: $\mathrm{T}(87)=4.87, \mathrm{P}<0.001)$. Therefore, $\mathrm{E}$-options' values elicited during the SP phase were more accurate than those elicited in the preceding ES-phase. This observation rules out forgetting as a plausible interpretation of the apparent experiential value neglect pattern observed in the ES phase.

\section{Ruling out generalization issues and assessing the robustness to practice}

The above-reported results from 4 experiments and 3 preference elicitation methods indicate that the experiential value neglect phenomenon cannot be accounted for by insufficient learning nor by mere forgetting. In the present section we rule out two additional alternative explanations. First, it should be noted that the ES phase involves a generalization process, because the E-options are extrapolated from the decision context where their subjective values are originally learned. It is therefore conceivable that the apparent experiential value neglect is spuriously created by a generalization problem. Second, in the previously reported experiments, participants went through the different phases (LE, ES and SP) only once: perhaps participants were somehow taken by surprise by the ES phase. In that case, presenting them different phases of the experiment twice will possibly allow them to improve their decisions by anticipating the ES-phase (36).

To control for generalization and practice, we run two additional experiments. In Exp. 5 and Exp. 6 ( $\mathrm{N}=71$ and $\mathrm{N}=66)$, after the learning phase, we interleaved the ES-choices with choices involving E-options presented in all possible combinations (referred to as EE-choices). Thus, in all cases except one, EE-choices required being able to generalize their value to new decision problems. As in ES-choices, we plotted the probability of choosing a given E-option as a function of the alternative E-option (Fig. 3B). To check whether experiential value neglect disappears if participants are given the opportunity to learn how to make ES decisions, Exp. 6 included a second session where we repeated all phases (LE, ES, ES and SP). Of note, E-options in the second sessions were materialized by a new set of symbols. 
A

Exp. 5

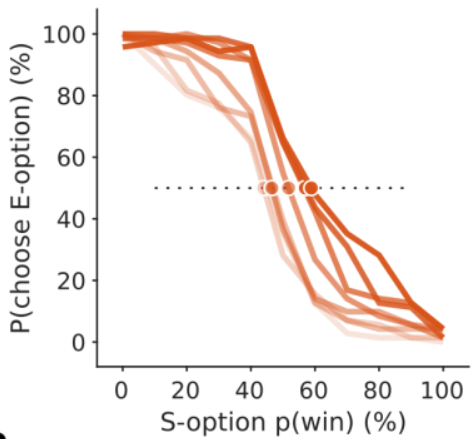

B
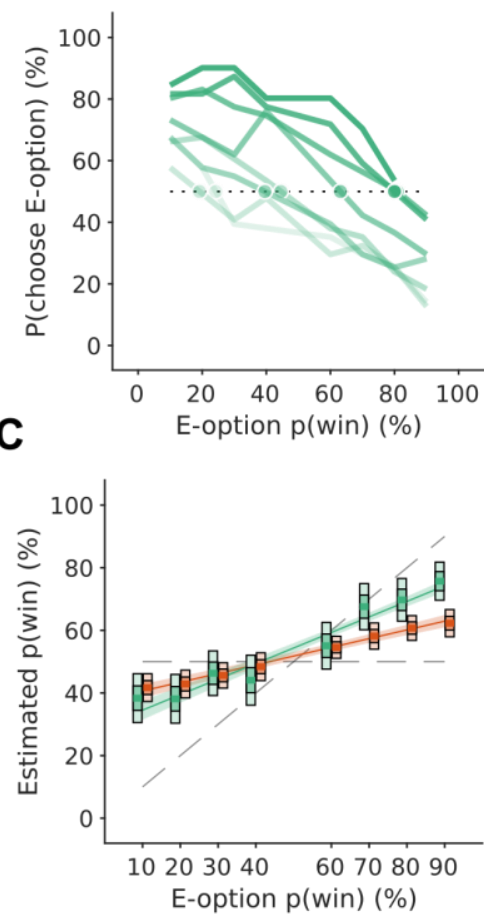

D

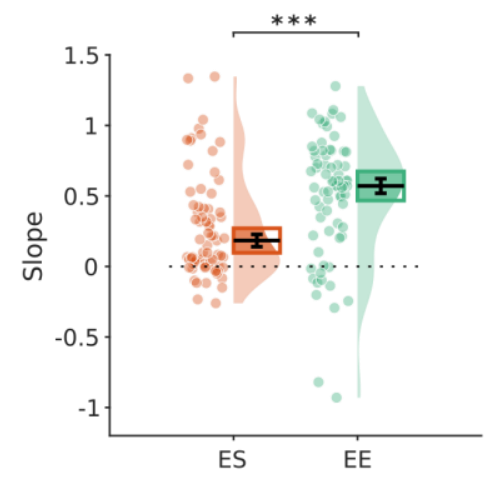

Exp. 6.1
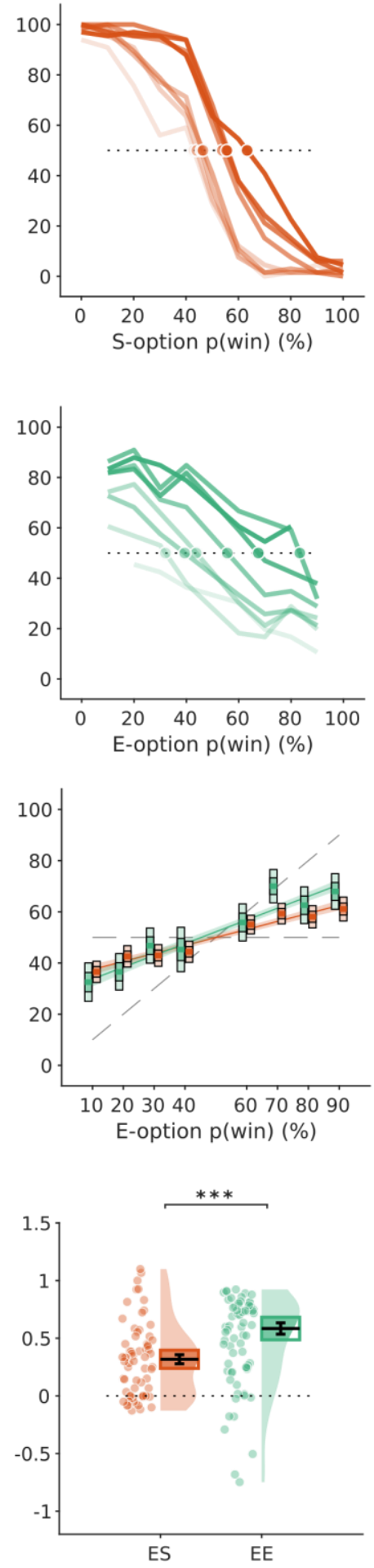

Experiential-Symbolic (ES)
Exp. 6.2
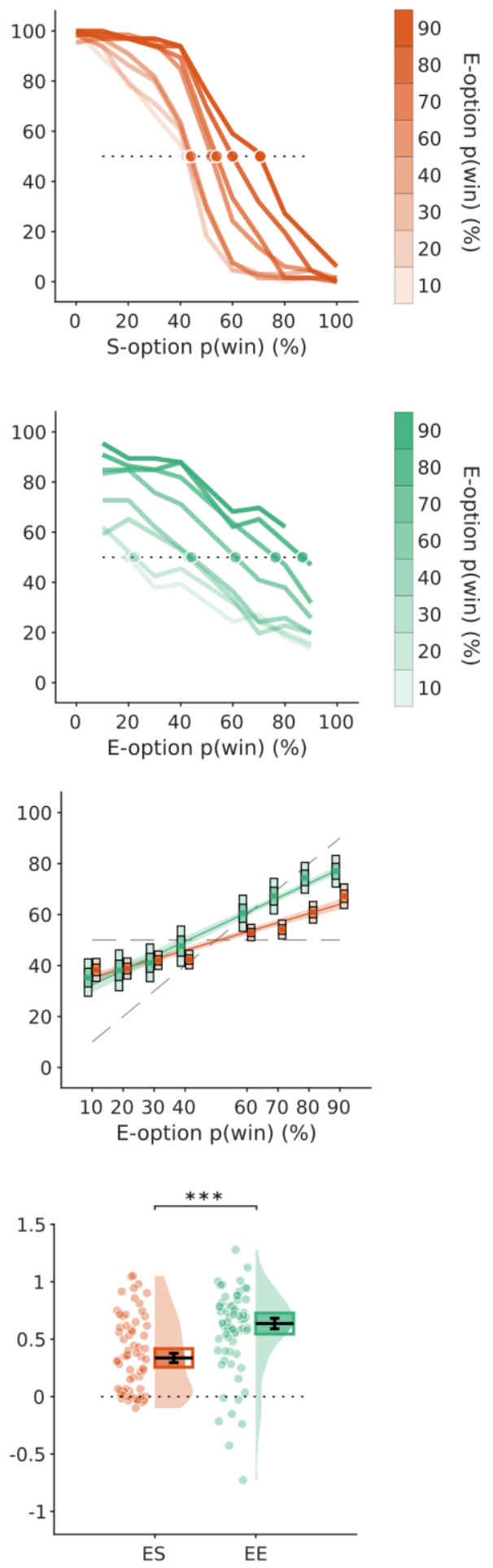

Experiential-Experiential (EE) 
Fig 3. Raw behavioral results and inferred option values in Experiments 5-to-6. (A) Average probability of choosing an E-option over a S-option during ES phase. The color of the curves indicates the value of the E-option (lowest: light orange; highest: dark orange). Dots represent the empirical indifference points, the value of a lottery that correspond to a probability of choosing the symbol $50 \%$ of the times. Exp. 6.1 and Exp. 6.2 refers to the first and the second session, respectively. (B) Average probability of choosing an E-option over another E-option during EE phase. The color of the curves indicates the value of the Eoption (lowest: light green; highest: dark green). Dots represent the empirical indifference points, the value of a lottery that corresponds to a probability of choosing the symbol $50 \%$ of the times. (C) The panels represent for each symbol the inferred value (as expressed by the probability of winning; $p($ win)) as a function of the actual value. ES estimates are represented in orange and EE estimates in green. In the data-boxes, the dark tone line represents the mean, mid-dark tone the standard mean error, light tone a $95 \%$ confidence interval. The lines represent linear regression (dark tone), and the average standard mean error (light tone). (D) Comparison of individual inferred slopes obtained from linear fit (see Fig. 3C) in two modalities (ES and EE in orange and green, respectively). The black lines represent mean and standard error of the mean. The colored boxes represent $95 \%$ confidence interval. The shaded area represents probability density functions. ${ }^{* * *} \mathrm{p}<0.001$ two sample $\mathrm{t}$-test.

EE-choices curves revealed that participants were capable of successfully extrapolating the value of the E-options to new decision problems involving other E-options. On the other side, the ES-choices were consistent with experiential values neglect, thus replicating the previous experiments (of note, the LE-phase of Exp. 5 and Exp. 6 presented the same characteristics as that of Exp. 3: complete feedback and block design) (Fig. 3A).

To formally assess the difference between EE- and ES-choices, we calculated for each participant their option-specific indifference points, following the same procedure used for ES-choices and we compared the inferred slopes across decision modalities. EE-inferred slopes were consistently significantly higher than ES slopes in both Exp. 5 and Exp. 6 (Exp. 5: $\mathrm{T}(70)=4.5, \mathrm{P}<0.001$; Exp. 6.1: $\mathrm{T}(65)=4.08, \mathrm{P}<0.001$ ).

Being presented with the whole experiment a second time had no detectable effect in choice behavior in neither the EE- or the ES-phase. Indeed, we observe no significant increase in the slopes in neither ES- $(\beta=0.04, \mathrm{~T}(260)=0.84, \mathrm{P}=0.4)$ nor $\mathrm{EE}$ - choices $(\beta=0.1, T(260)=1.59, P=0.11)$ and the $E S$-inferred slopes were still significantly smaller compared to EE- ones (Exp. 6.2: $\mathrm{T}(65)=5, \mathrm{P}<0.001$ ). This suggests that being exposed with the whole experiment one time and, by doing so giving participants the possibility to adjust the decision strategy does not affect the main results. 
A

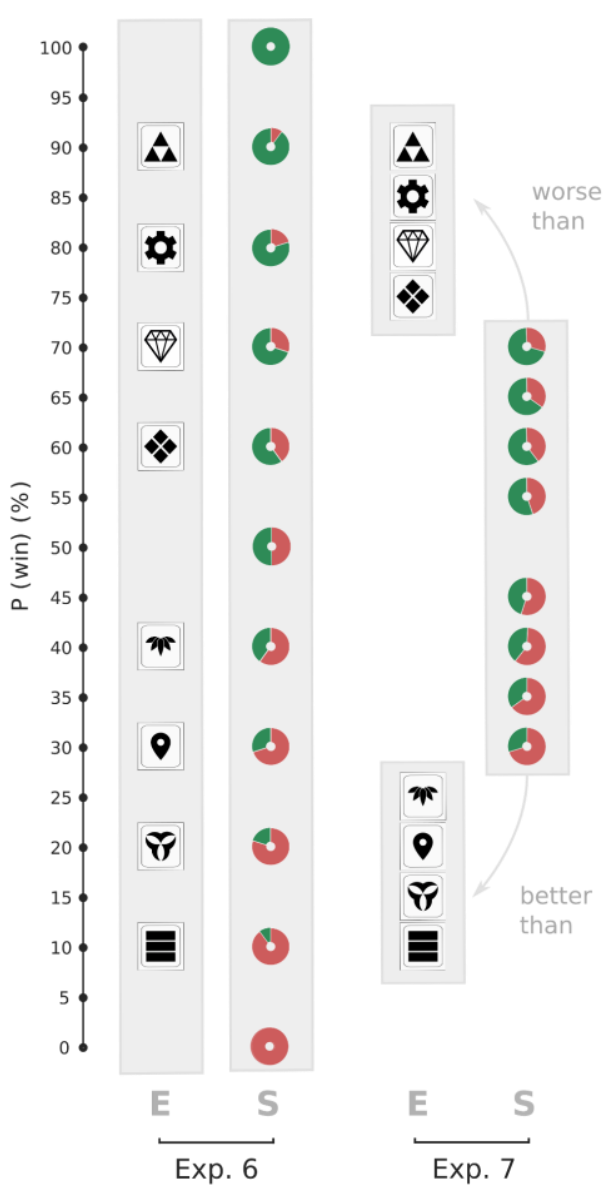

B

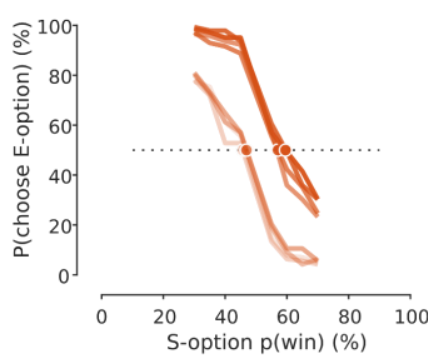

D

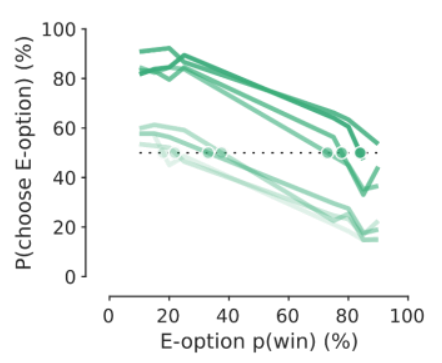

$\mathbf{F}$

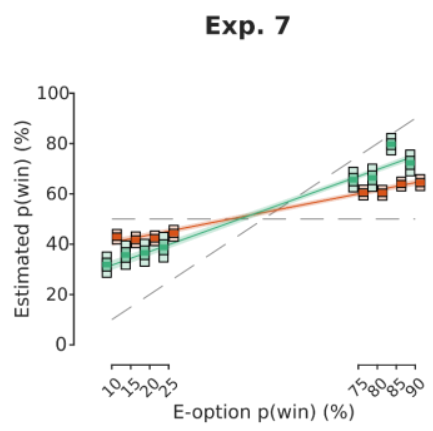

- Experiential neglect

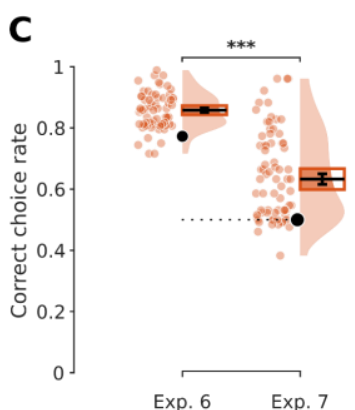

E
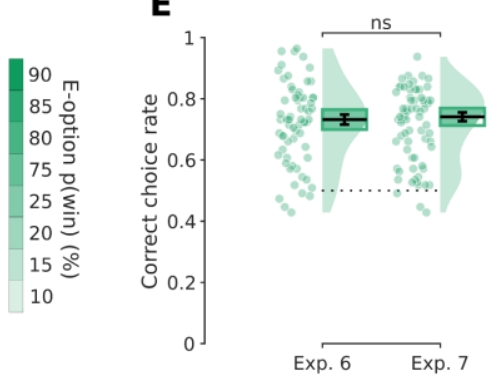

G

Exp. 7

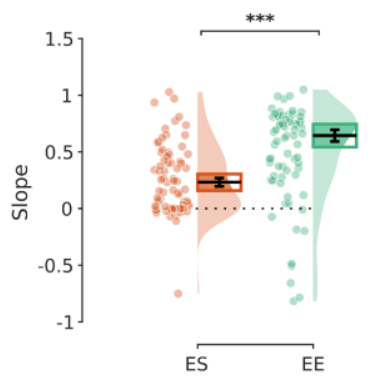

Fig 4. Option values and behavioral results in Experiment 7. (A) The panel shows and compare the options value in Exp .1-6 to that of Exp 7. In Exp. 7, we reorganized E-options and S-options values such that half of the E-options have higher expected-values than all S-options and, conversely the other half have lower expected-values. In such an arrangement, a participant fully neglecting the E-options values in the ES phase will end up with random choices in respect to utility maximization (B) Average probability of choosing an E-option over a S-option during ES phase. The color of the curves indicates the value of the E-option (lowest: light orange; highest: dark orange). Dots represent the empirical indifference points, the value of a lottery that correspond to a probability of choosing the symbol $50 \%$ of the times. (C) Expected value maximizing (i.e., correct) choices in the ES phase of Exp. 6 compared to Exp. 7. The black lines represent mean and standard error of the mean. The colored boxes represent $95 \%$ confidence interval. The shaded area probability represents density functions. ${ }^{* * *} p<0.001$ two-sample t-test. (D) Average probability of choosing an E-option over another E-option during EE phase. The color of the curves indicates the value of the E-option (lowest: light green; highest: dark green). Dots represent the empirical indifference points, the value of a lottery that correspond to a probability of choosing the symbol $50 \%$ of the times. (E) Expected value maximizing (i.e., correct) choices in the EE phase of Exp. 6 compared to Exp. 7. The black lines represent mean and standard error of the mean. The colored boxes represent $95 \%$ confidence interval. The shaded area probability density functions. ${ }^{* * *} \mathrm{p}<0.001$ two-sample t-test. $(\mathbf{F})$ The panel 
represents for each symbol the inferred value (as expressed by the probability of winning; $p($ win)) as a function of the actual value. ES estimates are represented in orange and EE estimates in green. In the data-boxes, the dark tone line represents the mean, mid-dark tone the standard mean error, light tone a $95 \%$ confidence interval. The lines represent linear regression (dark tone), and the average standard mean error (light tone). (G) Comparison of individual inferred slopes obtained from linear fit (see Fig. 4F) in two modalities (ES and EE; in orange and green, respectively). The black lines represent mean and standard error of the mean. The colored boxes represent $95 \%$ confidence interval. The shaded area probability represents density functions. ${ }^{* \star *} \mathrm{p}<0.001$ paired two-sample t-test.

\section{Experiential value neglect persists even when it bears an economic cost}

Analysis of choice behavior in the ES show that learned values of the E-options are largely neglected, as if participants were deciding on the basis of the value of the S-options only, and this despite the fact performance in the LE, SP and EE-choices indicate that E-option values are well learned and memorized. Neglecting experiential values seems, at least prima facie, suboptimal for the decision process, as taking into account all relevant information is considered a hallmark of normative behavior $(37,38)$. However, if E-option information processing (e.g. memory access/retrieval) is costly or if neglecting E-options does not hinders decision performance dramatically, it may become rational to do so (3941).

To evaluate this possibility, we simulated choices based on an extreme version of the experiential neglect rule: if an S-option has positive expected value, choose it, otherwise choose the E-option. These simulations show that, applied to the decision problems of the ES phase from experiments 1-to-6, extreme experiential neglect still generates $77 \%$ of expected-value maximizing choices. This result is actually not as counterintuitive as it initially appears: by design, a positive lottery is the most advantageous option in $\geq 50 \%$ of the decision problems in which it appears, and the converse is true for the negative expected value lotteries. These considerations suggest that, instead of representing an intrinsic cognitive limitation of value-based decision-making, the experiential value neglect is a rational heuristic strategy deployed by efficient (or lazy) decision-makers maximizing an accuracy-effort trade-off (42-45).

In order to test this new interpretation of the results, we designed a new experiment (Exp. 7) where we reorganized $\mathrm{E}$ - and S-options probabilities in a way that makes neglecting experiential values economically disadvantageous (Fig. 4A). In this new configuration, 
402 the narrower range of S-option values are nested within the broader E-option values, so 403 that any given S-option has a higher expected value compared to the 4 negative $\mathrm{E}$ 404 options, and a lower expected value compared to the 4 positive E-options. Such 405 configuration guarantees that participants neglecting E-option values in the ES-phase will 406 exhibit a chance-level choice accuracy (50\% of expected value maximizing choices). 407 Except for the modification of the lotteries, Exp. 6 present the exact number of trials.

408 Despite this stronger economic incentive, the behavioral pattern in ES-phase remained 409 consistent with the experiential value neglect scenario (Fig. 4B). The significant 410 difference between ES and EE slopes persisted in Exp. $7(T(70)=5.12, P<0.001)$, 411 suggesting that despite the reorganization of probabilities, we were still able to elicit more 412 accurate E-option values from EE-choices (Fig.4F, Fig. 4G). As a consequence, 413 compared to Exp. 6, the accuracy in the ES-choices significantly dropped in Exp. 7 by 414 approximately $20 \%(T(94.97)=11.01, P<.001$, Fig 4 C $)$. Of note, the accuracy in the EE415 choices remained the same (Fig. 4D, Fig. 4E), with no significant difference between the 416 two experiments $\left(T(131.77)=0.38, P=1, B^{10}=0.19\right)$.

417 These findings indicate that experience values are neglected even when it involves an 418 (economic) cost. Therefore, the results are consistent with the idea that the experiential 419 value neglect reflects a hard-coded feature of hybrid choices between experiential and 420 symbolic option, rather than being strategically deployed by the relative lack of incentive 421 in Exp. 1-6.

\section{Controlling for ambiguity aversion}

423 E-options may be deemed more ambiguous, because their outcome probability 424 distributions are inferred from finite samples and cannot been known with absolute 425 precision or certainty. Experiential value neglect cannot be accounted by a simple form 426 of ambiguity aversion (46-48), because E-options are generally preferred compared to 427 negative expected value S-options (i.e., there is no systematic bias against E-options). 428 Nonetheless, to assess whether the participant's attitude toward ambiguous lotteries 429 differed between experiential and symbolic options in a final experiment we included 430 choices with ambiguous lotteries (i.e., lotteries, whose value was hidden). The results 431 (presented in the Supplementary Materials and Figure S1) indicate that ambiguity 
A

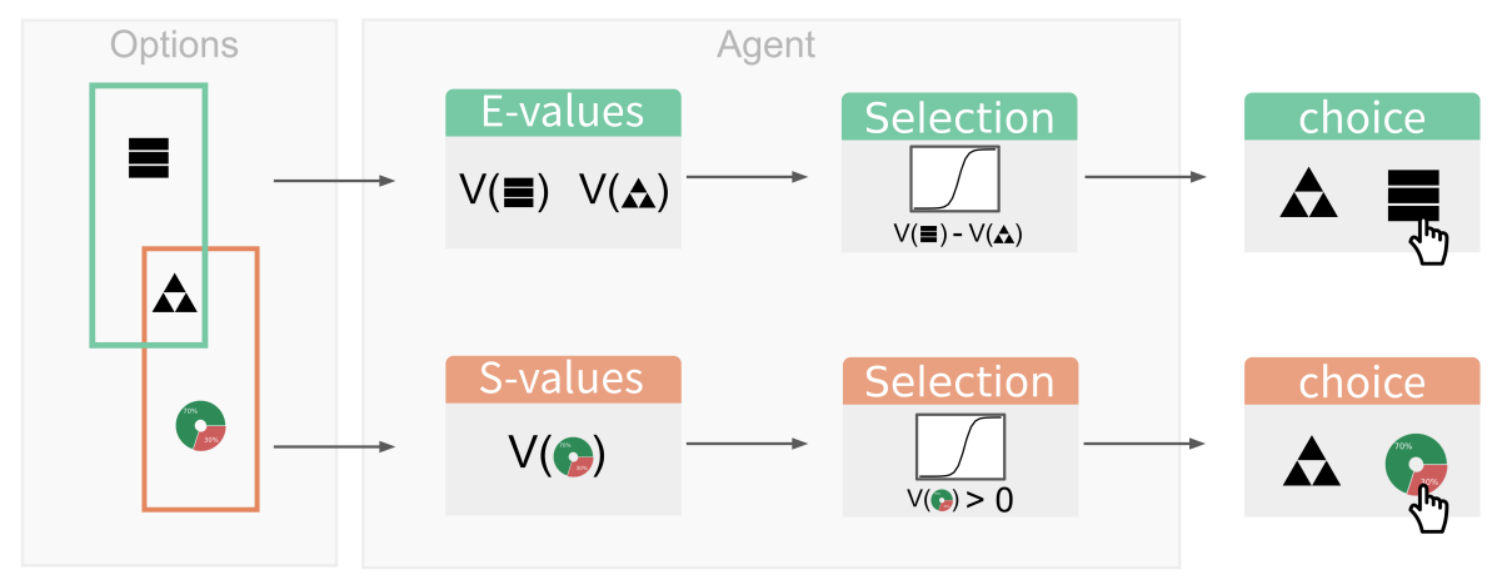

B

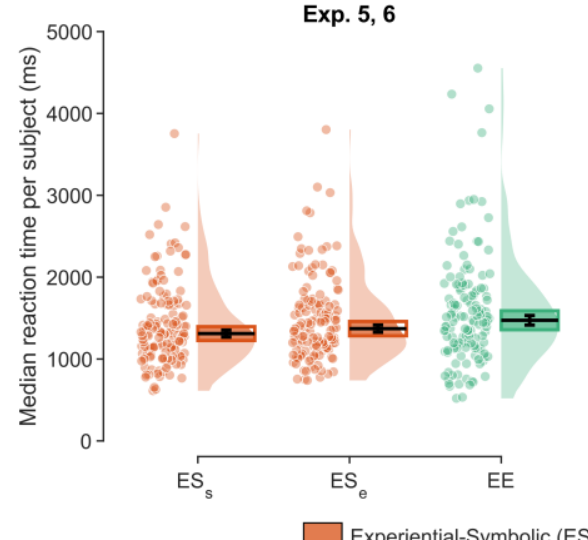

C

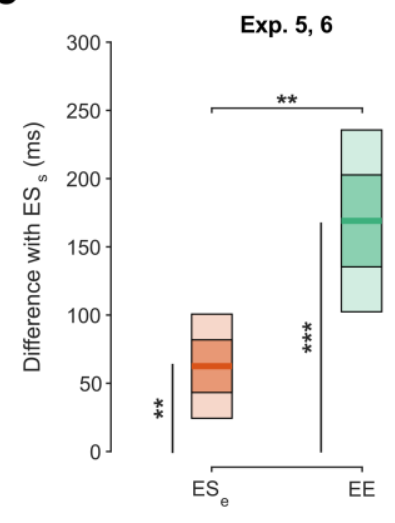

D

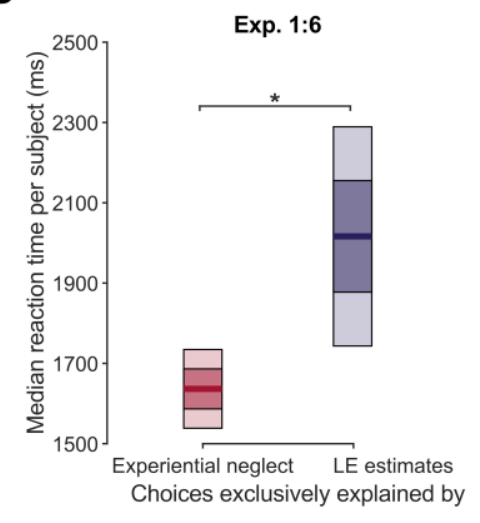

Fig. 5 Hypothetical decision model and reaction times analyses (A) The panel presents a schematic representation of the decision process in the EE- and the ES- phases, respectively. The two processes differ in that in the former case (EE) the decision is based by retrieving the values of both options, while in the latter case (ES), under an extreme form of experiential value neglect, only the value of the lottery matters. (B) Median reaction times across modalities. EE decisions are significantly longer than ES decisions (regardless of the choice taken in ES). When comparing when an S-option is chosen (ESs) and when an E-option is chosen (ESe) we also observed a significant difference. The black lines represent mean and standard error of the mean. The colored boxes represent $95 \%$ confidence interval. The shaded area probability density functions. (C) Different in reaction times differences $\left(E S_{e}-E S_{s}\right.$ in orange; $E E-E S_{s}$ in green). In the data-boxes, the dark tone line represents the mean, mid-dark tone the standard mean error, light tone a 95\% confidence interval. (D) Reaction times as a function of whether the ES-choices could be only explained by a total neglect of the experiential value (red) or whether they could only be explained by experiential values estimated from the learning phase (dark blue). In the data-boxes, the dark tone line represents the mean, mid-dark tone the standard mean error, light tone a $95 \%$ confidence interval. ${ }^{*} p<0.05$, 451 


\section{Reaction times analysis: a tale of two systems?}

453 Choice behavior differ across the ES- and the EE-choices. In the ES-phase, participants 454 neglect the experiential option value and to make choices only based on the symbolic 455 option value, so that, if the S-option is positive, it is chosen, otherwise it is rejected (Fig. 456 5A). On the other hand, EE-choices are based on the retrieval from memory of the 457 experiential values of both options. Thus, one decision process (ES-choices) seems to 458 involve the processing and representation of only one option value (the lottery), while the 459 other process (EE-choices) seems to involve the processing and the representation of 460 two option values. We hypothesized that these different processes translate into different 461 reaction times between the two choice modalities. To test this prediction, we compared 462 the reaction times in EE and ES-choices, while including only decisions with similar 463 objective value difference (49). Indeed, we found that ES decisions were faster compared 464 to EE decisions, both when the S-option is chosen - $\left(E S_{S}\right)$ and when the E-option is 465 chosen $-\left(E_{e}\right)(T(136)=6.02, \mathrm{P}<0.001 ; \mathrm{T}(136)=3.98, \mathrm{P}<0.001$; Fig. 5B and Fig. 5C) 466 Of note, within ES decisions, ES choices were also slightly but significantly slower the $467 \mathrm{ES}_{\mathrm{s}}$ choices $(\sim 50 \mathrm{~ms} ; \mathrm{T}(136)=4.35, \mathrm{P}<0.001)$, which may indicate that choosing the $\mathrm{E}-$ 468 option requires additional processing to retrieve and represent the value of the E-option. 469 To confirm this intuition, we considered two categories of ES-choices: choices exclusively 470 consistent with the participant choosing using the estimates inferred from the LE phase, 471 on one side, and choices consistent with a full experiential value neglect, on the other 472 side (Fig. S5). We observed that, in conformity with previous results, ES-choices that are 473 consistent with a full experiential value neglect are significantly faster than choices that 474 can only be explained taking into account the E-option values estimated from the LE475 phase $(\mathrm{T}(386)=2.27, \mathrm{P}<0.05)$ (Fig. 5D). Overall, the RT analyses support the idea that 476 choices based on the S-values of the lotteries required reduced cognitive processing 477 compared to those involving the retrieving from memory. Thus, E-values inferred from 478 ES-choices are consistent with the dual process model of Fig. 5A.

\section{Discussion}

480 Our results clearly indicate that the experiential and symbolic option values are not treated 481 symmetrically when making hybrid choices and speak against the idea of a central 
482 valuation system that encodes option values in a common currency, regardless of the 483 way they are built $(3,12)$. The key finding supporting this claim is provided by the analysis 484 of hybrid decision problems between experiential and symbolic cues, where choices 485 appeared to be made by largely neglecting value information acquired during the learning 486 phase. Crucially, by running several experiments and including multiple control measures, 487 we ruled out several alternative explanations for the experiential value neglect: this 488 decision-making pattern is not due to insufficient learning, forgetting, generalization issue, 489 or a lack of incentive. Finally, reaction time analyses are consistent with different 490 processing of experiential and symbolic values and with the idea of an additional cognitive cost associated with the memory retrieval of learned values. It seems that past 492 experiences and symbolic descriptions of possible outcomes ultimately generate value 493 representations different enough to make them largely incommensurable and that the 494 tension between the two is resolved by overweighting (or prioritizing) symbolic 495 information. In the following paragraphs we try to provide plausible reasons why these 496 values representations radically differ, why symbolic information is favored in hybrid 497 choices and which cognitive mechanisms could underlie the behavioral pattern observed.

498 Symbolic descriptions of lotteries in our task (and in general) involve separate information 499 about at least two different features of outcomes: payoffs (i.e., the amount of reward to 500 be won or lost) and their probability (50). Models of decision-making designed to explain 501 behavior in this kind of paradigms frequently assume that probability and payoffs are 502 processed individually. For instance, in prospect theory and its extensions, different 503 subjective weighting functions are supposed to apply to these variables $(51-53,14,54)$. 504 A separate representation of payoffs and probabilities is also assumed by models that do 505 not suppose the calculation of a multiplicative expected utility (55) and by models 506 supposing that decisions are underpinned by feature-by-feature comparisons (56-60). 507 On the contrary, experience-based choices, as instantiated by simple reinforcement 508 learning tasks, are usually modeled assuming that the decision-makers represents a 509 unique numeric value for each state-action pair. The decision-maker can 'look-up' in this 510 value matrix before making their choice and, once an outcome is obtained it partially 511 overwrites the 'cached' values previously stored in memory, so that they approximate the 512 average outcome (61). Option value representation is therefore structurally very different 
513 from that of description-based choices, because the relevant features (payoffs and 514 probabilities) are never explicitly represented as separate attributes of the outcomes. 515 Furthermore, some authors even suggest that reinforcement-based choices may bypass 516 the calculation of reward-based option-specific values, and is underpinned by what is 517 called direct policy learning (62-65). Our results seem to reject an extremely orthodox 518 interpretation of direct policy learning (accuracy in the learning phase was sensitive to the 519 value difference between options and experiential values were successfully generalized 520 to new combinations). It is nonetheless plausible to conceive that - at least to some extent 521 - reinforcement-based decisions involve a value-free (policy-based) component that can 522 be hardly compared with the subjective extracted from explicit payoffs and probabilities. 523 Functional neuroimaging investigations of experiential and symbolic decision-making 524 may also shed light on the debate about value representation across modalities. While 525 functional meta-analyses identified overlapping correlates of experiential and symbolic 526 values (17-20), the putative neural mechanisms of reinforcement-based and description527 based decisions differ in many crucial respects. First of all, the most influential and 528 consensual neural models of reinforcement-based leaning and decision-making give a 529 preponderant role to dopamine-induced neural plasticity circuits (66-68). More 530 specifically dopamine-dependent plasticity is supposed to drive action selection by 531 shaping the strength of the synapses between the frontal cortex and the basal ganglia $532(69,70)$. Current neural models do not attribute to dopamine-driven processes and the 533 basal ganglia a prominent role in description-based choices. Rather, they suppose that 534 the decision process is solved by cortical circuits (71-74), following an evidence 535 accumulation process similar to that observed for perceptual decisions $(75,76)$. Thus, 536 structural differences in the neural mechanisms of choices across modalities may 537 represent a biologically grounded bases of the representational difference between 538 experiential and symbolic values.

539 The representational tension of hybrid choices is solved by participants by neglecting the 540 experiential values and basing their choices on the symbolic value. Several control 541 analyses allowed us to formally exclude the possibility that this effect merely arise from 542 insufficient knowledge of the experiential values. Why is the symbolic information 543 preferred? We suggest two not-mutually exclusive explanations. One possibility is that 
544 experiential value estimates are perceived as less precise. Note here that precision 545 represents the uncertainty about the value estimate itself (48). Indeed, assuming 546 imperfect memory storage and retrieval, it is conceivable that experiential values are less 547 precise compared to symbolic ones that can be perfectly calculated (77). According to 548 this interpretation, participants would quasi-systemically prioritize the more precise 549 source of information for their choices $(47,48,78)$. Another possibility is that participants 550 prefer discarding experiential information not to incur the cost associated with the cost of 551 memory retrieval $(79,80)$. Reaction times analysis was overall consistent with this idea, 552 because choices involving the processing of the experiential values were generally slower 553 compared to those involving symbolic ones, even if balanced in objective difficulty (49). 554 This latter interpretation leaves open the possibility that if one makes memory retrieval 555 less costly, the behavioral pattern could be reversed (i.e., we would witness symbolic 556 value neglect). This could be possible for example after extensive training, once 557 experience-based choices are routinized (81) or, conversely, by making symbolic 558 information harder to decode. These are interesting possibilities to be explored by future 559 studies.

560 Finally, we speculate on the possible cognitive mechanisms underlying the experiential 561 value neglect phenomenon and we identify two plausible candidates. The first mechanism 562 involves 'bottom-up' attentional processes. It is well-documented that attentional focus 563 biases evidence accumulation in value based decision-making $(82,83)$. It is therefore 564 conceivable that an attentional bias toward symbolic options may result in prioritizing 565 described information and neglecting experiential one. The second possible mechanism 566 involves a 'top-down' heuristic process, according to which the calculation of individual 567 option values is hijacked by a deterministic decision rules (44). Of note, even if we 568 managed to demonstrate experiential value neglect in situations where it is 569 disadvantageous (experiment 7), it can nonetheless be argued that this decision rule is 570 overall adaptive, because computationally cheap and satisfying in most situations (see 571 experiments 1-6).

572 To conclude, our results add to the collection of behavioral anomalies showing that 573 values representations are inherently dependent on the way they are built, as it is 
574 postulated by the 'construction of preference' framework $(84,14,85)$. More specifically, 575 our findings pose serious challenges to the default assumption that values 576 representations are shared across different decision-making modalities, traditionally 577 referred to as experience- and description-based. The incommensurability between 578 experiential and symbolic values results in behaving as if discarding acquired information 579 and consequently entails suboptimal decisions. These findings are worth exploring 580 outside the experimental setting because many real-life decisions involve a tension 581 between an experiential and a symbolic component. 


\section{Acknowledgements}

584 The authors thank Aurélien Baillon for helpful comments. SP is supported by the Institut 585 de Recherche en Santé Publique (IRESP, grant number : 20ll138-00), the Agence 586 National de la Recherche (CogFinAgent: ANR-21-CE23-0002-02; RELATIVE: ANR-21587 CE37-0008-01; RANGE : ANR-21-CE28-0024-01) and the Ville de Paris (Emergence(s)). 588 ML is supported by an SNSF Ambizione grant (PZOOP3_174127) and an ERC Starting 589 Grant (948671). The article was prepared in the framework of a research grant funded by 590 the International Laboratory for Social Neuroscience of the Institute for Cognitive 591 Neuroscience HSE (RF Government grant No 075-15-2019-1930) and by the 592 Departement d'études cognitives (FrontCog ANR-17-EURE-0017). 


\section{References}

595 1. P. A. Samuelson, A Note on the Pure Theory of Consumer's Behaviour. Economica. 5, 61$596 \quad 71$ (1938).

597 2. J. Von Neumann, O. Morgenstern, Theory of games and economic behavior (Princeton $598 \quad$ University Press, Princeton, NJ, US, 1944), Theory of games and economic behavior.

599 3. A. Rangel, C. Camerer, P. R. Montague, A framework for studying the neurobiology of

4. R. J. Herrnstein, Relative and absolute strength of response as a function of frequency of

5. B. F. Skinner, Science and human behavior (Simon and Schuster, 1965).

6. R. S. Sutton, A. G. Barto, Reinforcement learning: An introduction (MIT press, 2018).

7. D. Bernoulli, Exposition of a New Theory on the Measurement of Risk. Econometrica. 22, 23-36 (1738).

8. P.-S. Laplace, Essai philosophique sur les probabilités (H. Remy, 1829).

9. P. Wakker, A. Tversky, An axiomatization of cumulative prospect theory. J. Risk Uncertain. 7, 147-175 (1993).

10. D. Kahneman, A. Tversky, in Handbook of the fundamentals of financial decision making: Part I (World Scientific, 2013), pp. 269-278.

612 11. B. De Martino, D. Kumaran, B. Seymour, R. J. Dolan, Frames, biases, and rational decision-making in the human brain. Science. 313, 684-687 (2006).

614 12. P. W. Glimcher, Foundations of neuroeconomic analysis (OUP USA, 2011).

615 13. C. F. Camerer, A review essay about Foundations of Neuroeconomic Analysis by Paul Glimcher. J. Econ. Lit. 51, 1155-82 (2013).

617 14. I. Vlaev, N. Chater, N. Stewart, G. D. A. Brown, Does the brain calculate value? Trends $618 \quad$ Cogn. Sci. 15, 546-554 (2011).

619 15. N. Stewart, EPS Prize Lecture: Decision by sampling: The role of the decision environment in risky choice. Q. J. Exp. Psychol. 62, 1041-1062 (2009).

16. I. Erev, E. Ert, O. Plonsky, D. Cohen, O. Cohen, From anomalies to forecasts: Toward a descriptive model of decisions under risk, under ambiguity, and from experience. Psychol. Rev. 124, 369 (2017).

17. O. Bartra, J. T. McGuire, J. W. Kable, The valuation system: a coordinate-based metaanalysis of BOLD fMRI experiments examining neural correlates of subjective value. Neuroimage. 76, 412-427 (2013). 
627

628

629

630

631

632

633

634

635

636

637

638

639

640

641

642

643

644

645

646

647

648

649

650

651

652

653

654

655

656

657

658

659

660

661

18. J. Garrison, B. Erdeniz, J. Done, Prediction error in reinforcement learning: a metaanalysis of neuroimaging studies. Neurosci. Biobehav. Rev. 37, 1297-1310 (2013).

19. J. A. Clithero, A. Rangel, Informatic parcellation of the network involved in the computation of subjective value. Soc. Cogn. Affect. Neurosci. 9, 1289-1302 (2014).

20. E. Fouragnan, C. Retzler, M. G. Philiastides, Separate neural representations of prediction error valence and surprise: Evidence from an fMRI meta-analysis. Hum. Brain Mapp. 39, 2887-2906 (2018).

21. R. Hertwig, I. Erev, The description-experience gap in risky choice. Trends Cogn. Sci. 13, 517-523 (2009).

22. C. R. Madan, E. A. Ludvig, M. L. Spetch, The role of memory in distinguishing risky decisions from experience and description. Q. J. Exp. Psychol. 70, 2048-2059 (2017).

23. D. U. Wulff, M. Mergenthaler-Canseco, R. Hertwig, A meta-analytic review of two modes of learning and the description-experience gap. Psychol. Bull. 144, 140 (2018).

24. B. Garcia, F. Cerrotti, S. Palminteri, The description-experience gap: a challenge for the neuroeconomics of decision-making under uncertainty. Philos. Trans. R. Soc. B. 376, 20190665 (2021).

25. D. Kellen, T. Pachur, R. Hertwig, How (in)variant are subjective representations of described and experienced risk and rewards? Cognition. 157, 126-138 (2016).

26. I. Erev, E. Ert, A. E. Roth, E. Haruvy, S. M. Herzog, R. Hau, R. Hertwig, T. Stewart, R. West, C. Lebiere, A choice prediction competition: Choices from experience and from description. J. Behav. Decis. Mak. 23, 15-47 (2010).

27. T. H. B. FitzGerald, B. Seymour, D. R. Bach, R. J. Dolan, Differentiable Neural Substrates for Learned and Described Value and Risk. Curr. Biol. 20, 1823-1829 (2010).

28. S. R. Heilbronner, B. Y. Hayden, The description-experience gap in risky choice in nonhuman primates. Psychon. Bull. Rev. 23, 593-600 (2016).

29. W. M. DuCharme, M. L. Donnell, Intrasubject comparison of four response modes for "subjective probability" assessment. Organ. Behav. Hum. Perform. 10, 108-117 (1973).

30. R. A. Rescorla, A theory of Pavlovian conditioning: Variations in the effectiveness of reinforcement and nonreinforcement. Curr. Res. Theory, 64-99 (1972).

31. T. E. J. Behrens, M. W. Woolrich, M. E. Walton, M. F. S. Rushworth, Learning the value of information in an uncertain world. Nat. Neurosci. 10, 1214-1221 (2007).

32. A. G. E. Collins, The Tortoise and the Hare: Interactions between Reinforcement Learning and Working Memory. J. Cogn. Neurosci. 30, 1422-1432 (2018).

33. S. Palminteri, M. Khamassi, M. Joffily, G. Coricelli, Contextual modulation of value signals in reward and punishment learning. Nat. Commun. 6, 1-14 (2015). 
662

663

664

665

666

667

668

669

670

671

672

673

674

675

676

677

678

679

680

681

682

683

684

685

686

687

688

689

690

691

692

693

694

34. S. Bavard, A. Rustichini, S. Palminteri, Two sides of the same coin: Beneficial and detrimental consequences of range adaptation in human reinforcement learning. Sci. Adv. 7, eabe0340.

35. G. M. Becker, M. H. DeGroot, J. Marschak, Measuring utility by a single-response sequential method. Behav. Sci. 9, 226-232 (1964).

36. J. Rieskamp, P. E. Otto, SSL: A Theory of How People Learn to Select Strategies. J. Exp. Psychol. Gen. 135, 207-236 (2006).

37. L. J. Savage, The foundations of statistics (Courier Corporation, 1972).

38. B. L. Lipman, Information Processing and Bounded Rationality: A Survey. Can. J. Econ. Rev. Can. Econ. 28, 42-67 (1995).

39. V. M. Chase, R. Hertwig, G. Gigerenzer, Visions of rationality. Trends Cogn. Sci. 2, 206214 (1998).

40. G. Gigerenzer, W. Gaissmaier, Heuristic decision making. Annu. Rev. Psychol. 62, 451482 (2011).

41. B. Mackowiak, F. Matejka, M. Wiederholt, Rational inattention: A review (2021).

42. H. A. Simon, Theories of bounded rationality. Decis. Organ. 1, 161-176 (1972).

43. H. A. Simon, A. Newell, Human problem solving: The state of the theory in 1970. Am. Psychol. 26, 145 (1971).

44. G. E. Gigerenzer, R. E. Hertwig, T. E. Pachur, Heuristics: The foundations of adaptive behavior. (Oxford University Press, 2011).

45. H. A. Simon, Administrative behavior (Simon and Schuster, 2013).

46. D. Ellsberg, Risk, Ambiguity, and the Savage Axioms. Q. J. Econ. 75, 643-669 (1961).

47. D. Frisch, J. Baron, Ambiguity and rationality. J. Behav. Decis. Mak. 1, 149-157 (1988).

48. C. Camerer, M. Weber, Recent developments in modeling preferences: Uncertainty and ambiguity. J. Risk Uncertain. 5, 325-370 (1992).

49. I. Krajbich, B. Bartling, T. Hare, E. Fehr, Rethinking fast and slow based on a critique of reaction-time reverse inference. Nat. Commun. 6, 7455 (2015).

50. C. A. Holt, S. K. Laury, Risk aversion and incentive effects. Am. Econ. Rev. 92, 1644-1655 (2002).

51. A. Tversky, D. Kahneman, Prospect theory: An analysis of decision under risk. Econometrica. 47, 263-291 (1979).

52. A. Tversky, D. Kahneman, Advances in prospect theory: Cumulative representation of uncertainty. J. Risk Uncertain. 5, 297-323 (1992). 
53. D. Prelec, The Probability Weighting Function. Econometrica. 66, 497 (1998).

54. J. Quiggin, Generalized expected utility theory: The rank-dependent model (Springer Science \& Business Media, 2012).

55. A. Soltani, E. Koechlin, Computational models of adaptive behavior and prefrontal cortex. Neuropsychopharmacology, 1-14 (2021).

56. K. Rayner, Eye movements in reading and information processing: 20 years of research. Psychol. Bull. 124, 372 (1998).

57. A. Glöckner, A.-K. Herbold, An eye-tracking study on information processing in risky decisions: Evidence for compensatory strategies based on automatic processes. J. Behav. Decis. Mak. 24, 71-98 (2011).

58. S. Fiedler, A. Glöckner, The Dynamics of Decision Making in Risky Choice: An EyeTracking Analysis. Front. Psychol. 3, 335 (2012).

59. V. Venkatraman, J. W. Payne, S. A. Huettel, An overall probability of winning heuristic for complex risky decisions: Choice and eye fixation evidence. Organ. Behav. Hum. Decis. Process. 125, 73-87 (2014).

60. J. A. Aimone, S. Ball, B. King-Casas, It's not what you see but how you see it: Using eyetracking to study the risky decision-making process. J. Neurosci. Psychol. Econ. 9, 137144 (2016).

61. R. S. Sutton, Learning to predict by the methods of temporal differences. Mach. Learn. 3, 9-44 (1988).

62. P. Dayan, L. F. Abbott, Theoretical neuroscience: computational and mathematical

63. J. Li, N. D. Daw, Signals in Human Striatum Are Appropriate for Policy Update Rather than modeling of neural systems (Computational Neuroscience Series, 2001).

64. B. Y. Hayden, Y. Niv, The case against economic values in the orbitofrontal cortex (or Value Prediction. J. Neurosci. 31, 5504-5511 (2011).

65. D. Bennett, Y. Niv, A. J. Langdon, Value-free reinforcement learning: policy optimization as

66. M. J. Frank, C. D'Lauro, T. Curran, Cross-task individual differences in error processing: neural, electrophysiological, and genetic components. Cogn. Affect. Behav. Neurosci. 7, 297-308 (2007).

67. A. G. Collins, M. J. Frank, Opponent actor learning (OpAL): modeling interactive effects of striatal dopamine on reinforcement learning and choice incentive. Psychol. Rev. 121, 337 (2014).

68. M. Möller, R. Bogacz, Learning the payoffs and costs of actions. PLOS Comput. Biol. 15, e1006285 (2019). 
731

732

733

734

735

736

737

738

739

740

741

742

743

744

745

746

747

748

749

750

751

752

753

754

755

756

757

758

759

760

761

762

763

764

765

69. P. Redgrave, T. J. Prescott, K. Gurney, The basal ganglia: a vertebrate solution to the selection problem? Neuroscience. 89, 1009-1023 (1999).

70. I. Bar-Gad, H. Bergman, Stepping out of the box: information processing in the neural networks of the basal ganglia. Curr. Opin. Neurobiol. 11, 689-695 (2001).

71. L. T. Hunt, N. Kolling, A. Soltani, M. W. Woolrich, M. F. Rushworth, T. E. Behrens, Mechanisms underlying cortical activity during value-guided choice. Nat. Neurosci. 15, 470-476 (2012).

72. A. Rustichini, C. Padoa-Schioppa, A neuro-computational model of economic decisions. J. Neurophysiol. 114, 1382-1398 (2015).

73. C. Padoa-Schioppa, K. E. Conen, Orbitofrontal cortex: a neural circuit for economic decisions. Neuron. 96, 736-754 (2017).

74. S. Farashahi, C. H. Donahue, P. Khorsand, H. Seo, D. Lee, A. Soltani, Metaplasticity as a Neural Substrate for Adaptive Learning and Choice under Uncertainty. Neuron. 94, 401414.e6 (2017).

75. A. C. Huk, M. N. Shadlen, Neural Activity in Macaque Parietal Cortex Reflects Temporal Integration of Visual Motion Signals during Perceptual Decision Making. J. Neurosci. 25, 10420-10436 (2005).

76. C. A. Hutcherson, B. Bushong, A. Rangel, A neurocomputational model of altruistic choice and its implications. Neuron. 87, 451-462 (2015).

77. C. Findling, N. Chopin, E. Koechlin, Imprecise neural computations as a source of adaptive behaviour in volatile environments. Nat. Hum. Behav. 5, 99-112 (2021).

78. R. Bricet, "Preferences for information precision under ambiguity" (THEMA (THéorie Economique, Modélisation et Applications), Université de ..., 2018).

79. R. Dukas, Costs of Memory: Ideas and Predictions. J. Theor. Biol. 197, 41-50 (1999).

80. H. Afrouzi, S. Kwon, Y. Ma, "A Model of Costly Recall" (working paper, Columbia University, 2020).

81. K. J. Miller, A. Shenhav, E. A. Ludvig, Habits without values. Psychol. Rev. 126, 292 (2019).

82. I. Krajbich, C. Armel, A. Rangel, Visual fixations and the computation and comparison of value in simple choice. Nat. Neurosci. 13, 1292-1298 (2010).

83. P. Sepulveda, M. Usher, N. Davies, A. A. Benson, P. Ortoleva, B. De Martino, Visual attention modulates the integration of goal-relevant evidence and not value. eLife. 9, e60705 (2020).

84. S. Lichtenstein, P. Slovic, The construction of preference (Cambridge University Press, 2006). 
766

767

768

769

770

771

772

773

774

775

776

777

778

779

780

781

782

783

784

785

786

787

788

789
85. B. Hayden, Y. Niv, The case against economic values in the brain (2020), doi:10.31234/osf.io/7hgup.

86. R. D. Luce, The choice axiom after twenty years. J. Math. Psychol. 15, 215-233 (1977).

87. R. D. Luce, Individual choice behavior: A theoretical analysis (Courier Corporation, 2012).

88. M. Lebreton, K. Bacily, S. Palminteri, J. B. Engelmann, Contextual influence on confidence judgments in human reinforcement learning. PLOS Comput. Biol. 15, e1006973 (2019).

89. R. C. Wilson, A. G. Collins, Ten simple rules for the computational modeling of behavioral data. eLife. 8, e49547 (2019). 
791 Methods and supplementary results

792 In this document we present the methods, as well as some additional results, including 793 those issued from an experiment (Exp. 8), which is only briefly mentioned in the main text.

\section{Experimental participants}

795 In total, we tested 787 participants (430 females; aged 31.09 \pm 10.42 years) distributed 796 across seven experiments. Participants were recruited via Prolific, a platform dedicated 797 to online research participants recruitment (https://prolific.co/). To assess participants' 798 engagement in the different tasks and their understanding of probability representation, 799 we inserted catch trials consisting in choices between two lotteries (S-options), with one 800 of the two cues being obviously better in terms of expected value maximization. In all 801 analyses we only retained the participants displaying $100 \%$ of correct choices in these 802 catch trials. In total 599 participants were included. Experiment 1 to 7 included the 803 following numbers of participants: 76,71, 83, 88, 71, 66, 71, 73 (see Table 1). Of note, 804 none of the results presented in the main or supplemental text was affected by the 805 exclusion of the participants.

\begin{tabular}{|lllllll|}
\hline Exp. & Outcome (LE) & Structure (LE) & Decision problems (LE) & Phases & Sessions & N \\
\hline 1 & partial & interleaved & 4 & LE ES SP & 1 & 76 \\
\hline 2 & partial & blocked & 4 & LE ES SP & 1 & 71 \\
\hline 3 & complete & blocked & 4 & LE ES SP & 1 & 83 \\
\hline 4 & complete & blocked & 2 & LE ES SP & 1 & 88 \\
\hline 5 & complete & blocked & 4 & LE ES EE SP & 1 & 71 \\
\hline 6 & complete & blocked & 4 & LE ES EE SP & 2 & 66 \\
\hline 7 & complete & blocked & 4 & LE ES EE SP & 2 & 71 \\
\hline 8 & complete & blocked & 4 & LE ES EE SP EA/SA & 2 & 73 \\
\hline
\end{tabular}

807 Table S1. Experiments parameters. The 'Exp.' column refers to the experiment number. The 'Outcome (LE)' column refers to the 808 outcomes displayed during a single LE phase trial. The column can take two values: partial (only obtained outcome) or complete (both 
obtained and forgone outcomes). The 'Structure (LE)' column refers to how the presentation of the options (or decision problems) was organized in the LE phase. 'Blocked' correspond to the case in which all trials belonging to a given option pair are presented in a row. Otherwise, when options pairs are distributed randomly, the value is set to 'interleaved'. The 'Decision problems (LE)' column refers to the number of option pairs presented in the LE phase. The 'Phases' column, refers to the specific phases present in a particular experiment. 'LE' refers to the learning phase. 'ES' stands for Experiential-Symbolic phase. 'EE' stands for Experiential-Experiential phase (performed after learning with no feedback). 'SP' stands for Stated Probability phase. 'EA/SA' stands for ExperientialAmbiguous and Symbolic-Ambiguous. The 'Sessions' column provides the number of sessions, i.e., how many times we repeated the sequence of phases with a different set of E-options. The ' $N$ ' column refers to the number of participants included in the experiment after exclusion of those displaying $>100 \%$ correct response rate in the ES catch trials.

The research was carried out following the principles and guidelines for experiments including human participants provided in the declaration of Helsinki (1964, revised in 2013). The INSERM Ethical Committee approved the study and participants provided written informed consent prior to their inclusion. To sustain motivation throughout the experiment, the tasks were economically incentivized. Specifically, in addition to a showup fee, participants were initially endowed with £2.5, and according to their choices, they could reach a maximum $£ 5$. The conversation rate was around $1 \mathrm{pt}=1$ cent and they were explained that all points won across the different phases were summed up. The average final bonus was $£ 4.05 \pm 0.72$, which was significantly higher compared to what they would have got in average following random choices $(T(615)=52.58, P<0.001)$.

\section{Data availability}

The analysis was performed using Matlab R2021a. The code and data for the analysis is available here: https://github.com/bsgarcia/RetrieveAndCompareAnalysis.

\section{Behavioral protocol}

The different experiments were conducted on a website programmed in javascript, html and css (code: https://github.com/bsgarcia/RetrieveAndCompare, testing: https://humanrl.scicog.fr/RandCTesting).

\section{Initial learning phase (LE phase)}

Participants first performed a probabilistic instrumental learning task (LE). Participants were provided with written instructions explaining that the aim of the task was to maximize 
840 their payoff by seeking monetary rewards and avoiding monetary losses. From 841 experiment 1 to 5, participants performed only one learning session. Experiment 6, 7 and 8428 for their part include 2 learning sessions. From experiment 1 to 7 , each learning session 843 contained four pairs of experiential cues (E-options), apart from experiment 4, which 844 contained 2 (but featured proportionally twice more trials). Each pair was fixed, so that a 845 given cue was always presented against the same other cue. Thus, within learning 846 sessions, pairs of cues represented stable choice contexts. Within each pair, the two cues 847 were associated to two outcomes; either winning a point $(+1)$ either losing one $(-1)$. The 848 four (two in experiment 4) cue pairs corresponded to four contexts of varying difficulty, 849 indexed by the difference in the probability of winning a point between the two cues. On 850 each trial, one pair was randomly presented with one cue on the right and the other on 851 left side of the screen. Participants were required to select, without time-limit, between 852 the two cues by left-clicking. After the choice, the selected cue was highlighted with a 853 black border while a transition effect was activated. The transition effect lasted approximately $1000 \mathrm{~ms}$ and revealed the outcome of the choice. The outcome was then displayed during approximately $1500 \mathrm{~ms}$. In experiments 1, 2, 3, 5, 6 and 7, the four pair of cues were presented 30 times each, for a total of 120 trials within sessions. In experiment 4 , the two pairs were presented 60 times each, to maintain an identical number of trials. In experiment 1, pairs of cues were presented in an interleaved manner, meaning they were distributed randomly across the 120 trials. From experiment 2 to 7 , pairs were presented in a blocked manner, meaning they were stacked in sequences of 30 choices.

862 Regarding feedback, there were two settings: partial and complete. A partial feedback 863 setting implied that only the outcome of the chosen option (or cue) was displayed, while 864 complete feedback means that both outcomes were displayed, regardless of the choice. 865 Experiment 1 and 2 involved partial feedback. From experiment 3 on, feedback was set 866 to complete.

\section{Hybrid choices between experiential and symbolic values (ES phase)}


869

870

871

872

873

874

875

876

877

878

879

880

881

882

883

884

885

886

887

888

889

890

891

892

893

894

After the LE phase, E-options were presented against symbolic cues (S-options). Soptions were implemented as pie-charts, where the green part indicates the probability to win a point, and the red part indicates the probability to lose a point. Each E-option (8) involved in the LE phase was presented against $11 \mathrm{~S}$-options (for a total of 88 trials), with probability of winning (and respectively loosing) a point ranging from $0 \%$ to $100 \%$, with a $10 \%$ step. On each trial, one pair was randomly presented with one cue on right and left side of the screen. Participants were required to select, without time-limit, between the two cues by left-clicking. After the choice, the selected cue was highlighted with a black border and the transition to the next trial, lasted approximately $1000 \mathrm{~ms}$. No feedback was presented during the ES phase. Participants were informed about their earnings only at the end.

Although the outcome was not displayed, participants were told that this phase was still incentivized, such that choice accuracy affected their bonus compensation.

\section{Assessing generalization of experiential values (EE phase)}

This phase is present in Experiment 5 to 8 . After the LE phase, each E-option was presented against other E-options. With 8 cues presented in the LE phase, it follows that each E-option was presented against the other $7 \mathrm{E}$-options, so that this phase contained 56 trials. EE choices were presented in the same time as the ES choices, because we wanted to avoid having them differ in terms of time elapsed since the LE phase. Thus, technically the EE and the ES phases overlap.

For each trial, one pair was randomly presented with one cue on the right and left sides of the screen. Participants were required to select, without time-limit, between the two cues by left-clicking. After the choice, the selected cue was highlighted with a black border and the transition to the next trial, lasted approximately $1000 \mathrm{~ms}$. The transition effect lasted approximately $1000 \mathrm{~ms}$ and leave place for the next trial. No feedback was presented. 
895

896

897

898

899

900

901

902

903

904

905

906

907

908

909

910

911

912

913

914

915

916

917

918

919

920

921

922

Although the outcome was not displayed, participants were told that they could still win (and lose) points during this phase, this phase was still incentivized, such that choice accuracy affected their bonus compensation.

\section{Stated Probability assessment (SP phase)}

In all experiments, participants were asked, for each E-option previously faced in the LE phase, the following question «What are the odds this symbol gives $a+1$ ?». They had to provide their answer on rating-scale, going from $0 \%$ to $100 \%$ with a $5 \%$ step.

Answers were incentivized via a matching probability procedure that is based on the Matching Probability Mechanism (1). More precisely, participant chose a probability ( $p$ ) for the presented E-option. A number $(r)$ is then randomly drawn in the interval [0 1]. If $p$ $>r$, the outcome of the choice was obtained using the E-option probability of winning and losing a point (as-if the E-option was chosen in the LE phase for instance). Otherwise, if $p<r$, the participant has $r(\%)$ chance of winning a point, and respectively $1-r(\%)$ chance of losing a point.

In other words, the higher the response $(p)$ of the participant, the higher the chances were the outcome would be determined by the E-option. Conversely, the lower the response (p), the higher the chances were that the outcome would be determined by the random lottery number $(r)$.

\section{Ambiguity assessment}

Preference towards ambiguous lotteries was assessed only in Experiment 8. After the LE phase, E-options as well as S-options were presented against an ambiguous cue. This ambiguous cue was represented by a greyed pie-chart, with a question mark on top. Consequently, it was represented similarly to S-options, i.e., as a lottery, which was however $100 \%$ ambiguous in the sense that it conveys no a priori information regarding probabilities of gains or losses (see Figure S1). Each E-option (8) and S-options (8), were presented against this ambiguous cue two times, resulting in a total of 32 trials. For each trial, one pair was randomly presented with one cue on the right and left sides of the 
923 screen. Participants were required to select, without time-limit, between the two cues by 924 left-clicking. After the choice, the selected cue was highlighted with a black border while 925 a transition effect was activated. The transition effect lasted approximately $1000 \mathrm{~ms}$ and 926 left room for the next trial. No feedback was presented.

927 Although the outcome was not displayed, participants were told that they still could win 928 (and lose) points during this phase, and that correct choices (i.e., choices maximizing 929 expected value) and wrong choices would thus affect their bonus compensation.

\section{Statistical and computational modeling}

\section{Inferential statistics}

932 All t-tests were realized using Python 3.9 and the pairwise_ttests function from the 933 pingouin library. Bonferroni's corrections were applied systematically. Linear regressions 934 were realized using Matlab R2020a fitlm function.

\section{E-option probabilities inference in ES and EE phases}

936 To infer a probability estimate (or indifference point) for each E-option from EE and ES 937 choices we proceeded as follows. In those phases, an E-option was assessed relatively 938 to other cues (either S-options, either other E-options). In the ES phase $11 \mathrm{~S}$-options were 939 presented against each E-option. In the EE phase $7 \mathrm{E}$-options were presented against 940 each E-option. Choosing the E-option that was currently assessed is always coded as 1 , 941 whereas choosing the cue presented against (an S-option in ES, or an E-option in EE) is 942 always coded as 0.

943 We note $c_{i}^{t} \in\{0,1\}$ the choice of a participant $i$ at trial $t$. Thus, for each E-option $j$ we 944 obtain a vector of choices $C_{i}^{j}=\left(c_{i}^{1}, c_{i}^{2}, c_{i}^{3}, \ldots c_{i}^{n}\right)$, with $n=11$ in the ES phase, and $n=7$ 945 in the EE phase. We then fit the following logistic function $(2,3)$ :

$$
f\left(C_{i}^{j}\right)=\frac{1}{1+e^{\beta_{i}\left(C_{i}^{j}-\lambda_{i}^{j}\right)}}
$$

947 With $\beta_{i}>0$ (which controls the slope of the function) being a free parameter unique to 948 each individual $i$, while $\lambda_{i}^{j} \in[0,1]$ (the function midpoint) is a free parameter that is 
949 estimated for each E-option $j$ and individual $i$. The indifference point $\lambda_{i}^{j}$ represents here

950 the probability where a preference shift (from one cue to another) occurs, and is thus a 951 subjective probability (or value) estimate for the E-option $j$ and participant $i$. Both 952 parameters were estimated through minimum negative log-likelihood estimation, using 953 matlab's fmincon function.

\section{Inferring E-option value estimated in the LE phase}

955 To infer E-option values in the learning (LE) phase, we fitted a reinforcement learning 956 model (or Q-learning model) to our data $(4,5)$.

957 The model treats each pair of cues as a state $s$. After a choice, each cue subjective 958 probability of winning a point $\left(p_{\text {win }}\right)$ was incrementally updated with the following 959 Rescorla-Wagner rule:

$$
p_{\text {win }}(s, c) \leftarrow p_{\text {win }}(s, c)+\alpha \delta_{c}
$$$$
p_{\text {win }}(s, u) \leftarrow p_{\text {win }}(s, u)+\alpha \delta_{u}
$$

Where $\alpha$ is the learning rate (which controls to what extent new information overrides previous one) for the chosen cue $(c)$ as well as the unchosen cue $(u)$. The associated prediction errors $\delta_{c}$ and $\delta_{u}$ are computed as follows:

$$
\delta_{c}=R_{c}-p_{\text {win }}(s, c)
$$

$$
\delta_{u}=R_{u}-p_{\text {win }}(s, u)
$$

967 Where $R_{c}$ and $R_{u}$ are the outcomes displayed for both chosen and unchosen cues. $R_{x}$ 968 took value of 1 , when the outcome was +1 pt, and 0 otherwise. Initial were set at 0.5 for 969 all options. Please note that for experiments 1 and 2, where only $R_{C}$ was displayed (partial 970 feedback setting) only $p_{\text {win }}(s, c)$ was updated. Decision was modeled using a softmax 971 function, where the actual probability of choosing a cue $a$ when presented against a cue $972 b$ was calculated as follows:

$$
P(s, a)=\frac{1}{1+e^{\beta\left(p_{\text {win }}(s, b)-p_{\text {win }}(s, a)\right)}}
$$


974 With $\beta>0$ being the temperature parameter, that implements choice stochasticity. As $\beta$ 975 decreases, the events of choosing $a$ or $b$ tend to become equi-probable. As $\beta$ increases, 976 the difference between $p_{\text {win }}(s, a)$ and $p_{\text {win }}(s, b)$ is amplified, and the choice becomes 977 more and more deterministic (until the function almost acts as an argmax policy).

\section{Model fitting}

979 Learning rate and temperature parameters (here denoted $\theta$ ) involved in the reinforcement 980 learning model were estimated by finding values that minimized the negative logarithm of 981 the posterior probability over the free parameters $(-\log (P(\theta \mid D))$, which was computed 982 as follows:

$$
-\log (P(\theta \mid D)) \propto-\log (P(D \mid \theta))-\log (P(\theta))
$$

984 Where $P(D \mid \theta)$ is likelihood of the data (i.e., the observed choices during the LE phase) 985 given certain parameter values, and $P(\theta)$ is the prior probability of those parameter 986 values.

987 The prior probability distribution over the learning rates was assumed as beta distributed 988 and quasi-uniform (betapdf(1.1, 1.1)). The softmax temperature was for its part assumed 989 to be gamma distributed (gampdf(1.2, 5)).

990 The optimization procedure was again performed using Matlab's fmincon function and 991 previously described in Lebreton et al., 2019.

\section{Parameter and choice recovery in EE and ES phases}

993 To quantify and statistically compare the differences in preferences observed in the ES 994 and EE phase, we estimated for each E-option its theoretical subjective value (expressed 995 in terms of probability of winning a point). This value is itself inferred in term of indifference 996 points. For instance, in ES choices, one E-option with $80 \%$ chance of winning will be 997 compared to range of S-options (going from $0 \%$ to $100 \%$ chance of winning a point). The 998 indifference point for the E-option considered is then the S-option value at which a 999 preference shift occurs between the two kinds of options (let's say, when the S-option is 1000 above $80 \%$, considering the decision-maker is rational). To infer those indifference points, 
1001 we fitted a logistic function to each subject choice history for each E-options in both EE 1002 and ES phases (see the methods section). We treated these indifference points as 1003 proxies for subjective values, i.e., E-option value estimates (or probability estimates, as 1004 in the numerical space considered they are equivalent).

1005 To assert that this fitting procedure is robust, and that we do not elicit random subjective 1006 values, we followed a parameter recovery procedure (7).

1007 We simulated EE and ES choices based on the (EE and ES) E-option value estimated 1008 from experiment 1 to 6.

1009 More precisely, for each subject, we simulated an agent going through its choice history, 1010 and we used the 8 inferred estimates (one for each E-option, each subject having its own 10118 indifferent points) to simulate new choices.

1012 We generate these choices using an argmax decision rule, meaning that the agent 1013 systematically selects the option with the highest value. Of note, in the simulated ES 1014 phase, we do not suppose any subjective deformation regarding S-options, such that the 1015 agent is directly informed of the objective expected-value to make its decision.

1016 At this point of the procedure, simulated choices can be compared to choices from 1017 behavioral data. By doing so, we can see how well they match, and therefore whether 1018 our value estimates allow us to correctly recover the choices actually made by our 1019 participants. EE choices are recovered up to 83\%, whereas ES choices are almost 1020 perfectly recovered, with a score of $96 \%$. The fact that EE choices are less recovered is 1021 not surprising, as the E-option estimates results from the comparison of one option 1022 against 7 others, when in the ES phase an E-option is presented against a wider range 1023 of alternative options (11), hence allowing better precision in the fitting of E-option value 1024 estimates.

1025 We then generate new E-option value estimates, by applying our initial logistic fitting 1026 procedure (see methods section) to this newly simulated data. We observe that E-option 1027 value estimates are almost perfectly recovered, both in the ES (Fig. S2) and EE (Fig. S3) 1028 phase, with a spearman $\rho$ that is systematically higher than .97. 

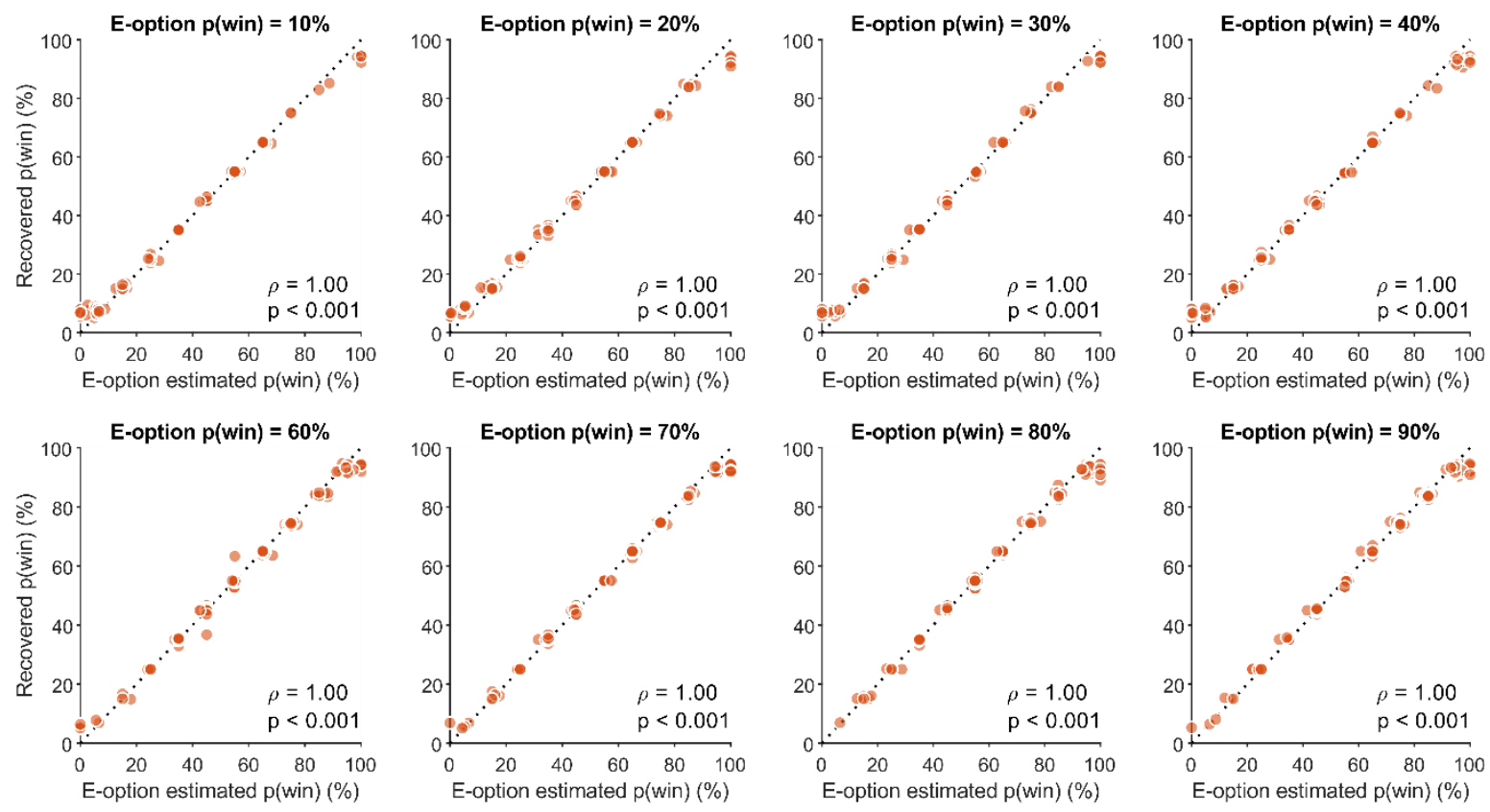

Fig. S2 Recovery of E-option estimated probabilities in Experiments 1-to-6, ES phase. We estimate 8 E-option value for each subject in the ES phase. Thereafter, going through each individual choice history, we simulate a new choice dataset using these value estimates as an input for an argmax decision rule. We apply our logistic fitting procedure again (see the methods section) on this simulated data, to generate new estimates. Then we run a spearman data. The grey dotted line corresponds to a perfect recovery of E-option probability estimates. 

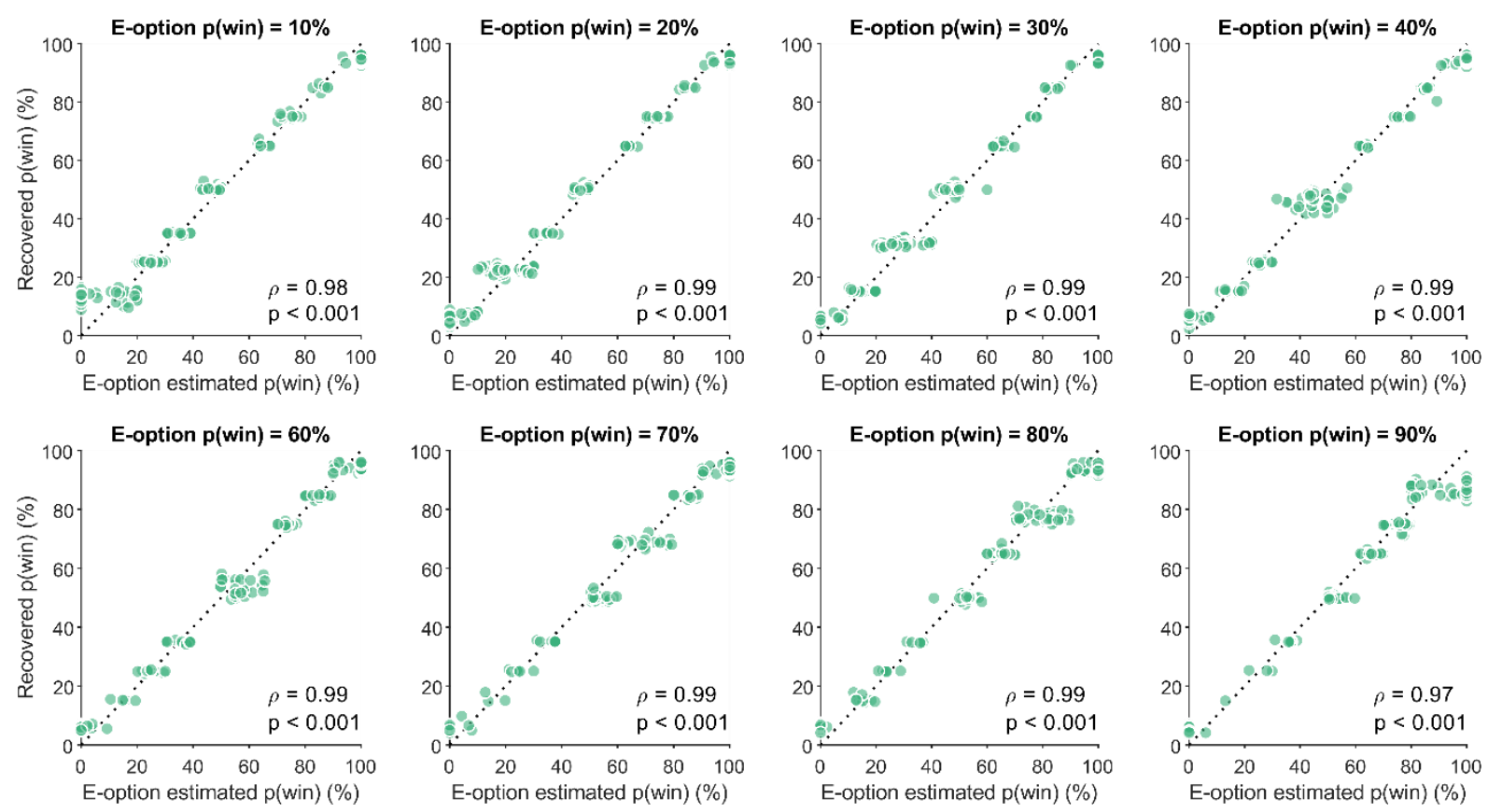

Fig S3. Recovery of E-option estimated probabilities in Experiments 1-to-6, EE phase. We estimate 8 E-option value for each subject in the EE phase. Thereafter, going through each individual choice history, we simulate a new choice dataset using these value estimates as input for an argmax decision rule. We apply our logistic fitting procedure again (see the methods section) on this simulated data, to generate new estimates. Then we run a spearman correlation to test the relationship between the estimates from the behavioral data and the estimates from the simulated data. The grey dotted line corresponds to a perfect recovery of E-option probability estimates. 
1046 Supplementary results

\section{Experiment 8}

1048 We devised Experiment 8 to test whether the behavioral pattern observed in the 1049 Experiential-Symbolic phase was the result of ambiguity aversion (8), i.e. that the 1050 participant have a preference for options with known probability distributions over option 1051 with unknown probability distributions. In other words, participants would neglect 1052 experiential expected-values estimated during the LE phase because they are reluctant 1053 toward ambiguous options (E-options) and consequently mainly rely on options actually providing full probabilistic information (S-options).

1055 1056 1057 1058 1059 1060 1061 1062 1063 1064 1065

1066 1067 1068 1069 1070

Thus, we presented each E-option (Fig. S1A: top) and S-option (Fig. S1A: bottom) against one ambiguous option (A-option), represented by a greyed pie-chart which conveyed no a priori information. Interestingly, the indifference point inferred for the Aoption was close to $50 \%$ (both when the A-option is presented against $\mathrm{E}$ - and A-options). It suggests that without a priori information, participants associate a $50 \%$ subjective probability of winning a point to the A-option. When presented against E-options (Fig. S1B: top), the A-option is preferred against E-option which probability of winning a point is inferior to $50 \%$, which suggests that those options are remembered as giving a negative expected-value. The preference is reversed when the E-option probability is above $50 \%$, showing that participants associate those options to positive expected-values. When presented against S-options (Fig. S1B: bottom)

Of note, E-options cannot be conflated with A-options for two reasons. First, when presented against A-options, E-options choice frequency increases monotonically with their associated objective probabilities. This result suggests that E-options are robustly linked to past outcome information, when it comes to comparing them to ambiguous stimuli.

Second, the experiential neglect pattern cannot be the result of pure ambiguity aversion, as E-options are in average preferred against S-options when the latter has a negative expected-value, regardless of the E-option value. It suggests that this preference for 
1074 known risks only holds in the gain domain, which excludes a pure preference toward 1075 known risks, i.e., a pure ambiguity aversion.

\section{A}
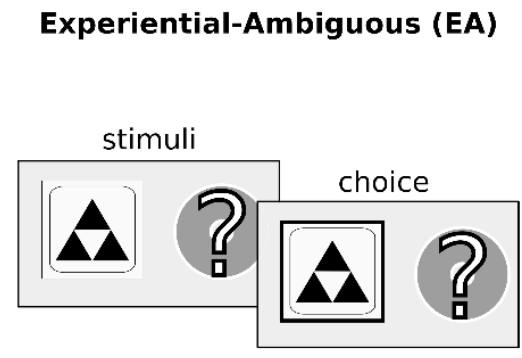

Symbolic-Ambiguous (SA)

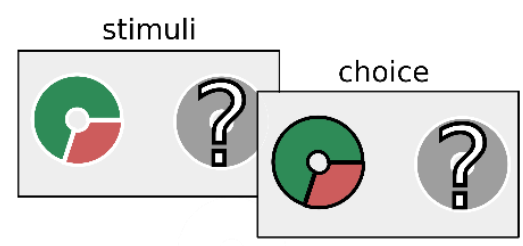

B

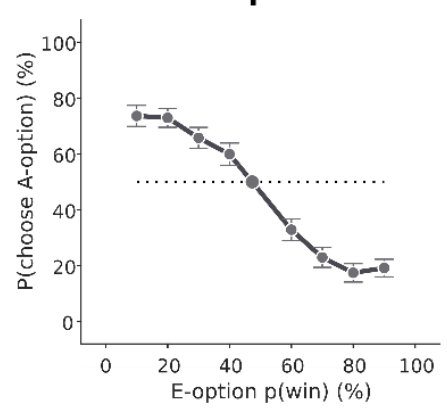

Exp. 8

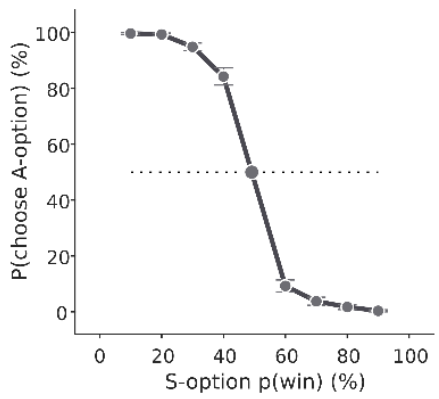

C

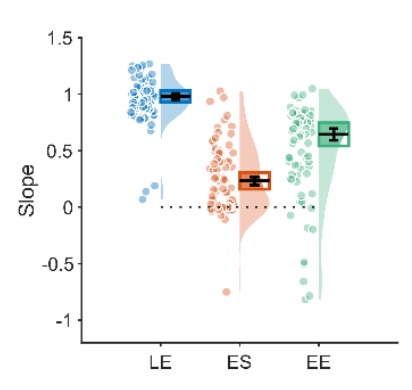

Exp. 8.2

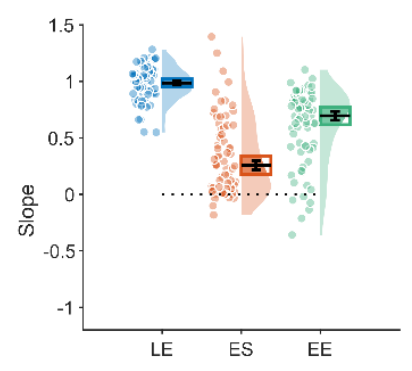

Fig. S1 Raw behavioral results and inferred option values in Experiments 8. (A) The topmost panel displays successive screens of a typical trial in the Experiential-Ambiguous (EA) phase. The bottommost panel displays successive screens of a typical trials in the Symbolic-Ambiguous (SA) phase. The EA-phase consists in binary choices between a symbol previous encountered in the LE-phase, and an ambiguous lottery (materialized as greyed pie-chart with a question mark on top). The SA-phase consists in binary choices between an explicit lottery (materialized as a pie-chart partly green for gain probabilities, and partly red for loss probabilities) and an ambiguous lottery (materialized as greyed pie-chart with a question mark on top). (B) Average probability of choosing an ambiguous option (A-option) over a E-option (top) or an Soption (bottom) during the ambiguity phase. Dots represent the empirical choice frequency of the A-option. The largest dot at the intersection of the grey dotted line represents the indifference point, i.e., when the subject chooses randomly between the two options. The error bars represent the standard error of the mean. (C) Comparison of individual inferred slopes obtained from linear fit in three modalities (LE, ES and $\mathrm{EE}$ in blue, orange and green, respectively). The black lines represent mean and standard error of the mean. The colored boxes represent $95 \%$ confidence interval. The shaded area represents the probability density function. ${ }^{* * *} \mathrm{p}<0.001$ paired sample t-tests.

Of note, introducing A-options among the other post-learning assessments did not affect the previously observed relation in inferred slopes (Fig. S1C). LE-inferred slopes were consistently significantly higher than ES slopes (Exp. 8.1: T(72)=13.05, $\mathrm{P}<0.001$; Exp 
1096

1097

1098

1099

1100

1101

1102

1103

1104

1105

1106

1107

1108

1109

1110

1111

1112

1113

1114

1115

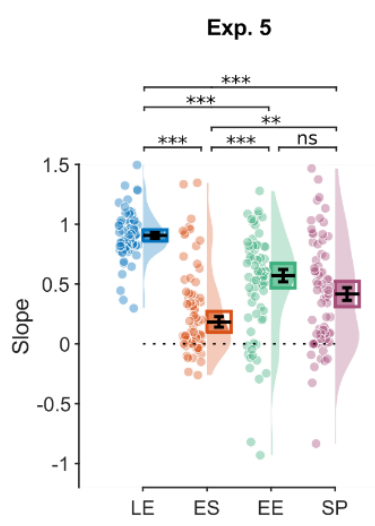

1116

8.2: $\mathrm{T}(72)=10.41, \mathrm{P}<0.001)$ as well as EE slopes (Exp. 8.1: $\mathrm{T}(72)=5.9, \mathrm{P}<0.001$; $\operatorname{Exp}$ 8.2: $\mathrm{T}(72)=6.38, \mathrm{P}<0.001)$. EE-inferred slopes for their part were systematically higher than ES slopes (Exp. 8.1: $\mathrm{T}(72)=5.78, \mathrm{P}<0.001 ; \operatorname{Exp} 8.2$ : $\mathrm{T}(72)=4.61, \mathrm{P}<0.001)$.

\section{Slope comparison among Experiments 5-to-8.}

From experiments 5-to-8 (Fig. S4), LE slopes were consistently and significantly higher than ES slopes (Exp. 5: $\mathrm{T}(70)=12.94, \mathrm{P}<0.001$; Exp. 6.2: $\mathrm{T}(65)=10.59, \mathrm{P}<0.001$; Exp. 7.2: $\mathrm{T}(70)=14.4, \mathrm{P}<0.001$;Exp. 8.2: $\mathrm{T}(72)=10.41, \mathrm{P}<0.001$ ), $\mathrm{E} E$ slopes (Exp. 5: $\mathrm{T}(70)=7.7, \mathrm{P}<0.001$; Exp. 6.2: $\mathrm{T}(65)=5.72, \mathrm{P}<0.001$; Exp. 7.2: $\mathrm{T}(70)=8.18, \mathrm{P}<0.001$;Exp. 8.2: $\mathrm{T}(72)=6.38, \mathrm{P}<0.001$ ), as well as SP (Exp. 5: $\mathrm{T}(70)=8.98, \mathrm{P}<0.001$; Exp. 6.2: $\mathrm{T}(65)=6.18, \mathrm{P}<0.001$; Exp. 7.2: $\mathrm{T}(70)=10.88, \mathrm{P}<0.001$;Exp. 8.2: $\mathrm{T}(72)=7.71, \mathrm{P}<0.001)$.

The EE slopes were the second closest to 1, i.e., the second closest to E-option objective values. They are consistently higher than ES slopes (Exp. 5: T(70)=4.48, $\mathrm{P}<0.001$; Exp. 6.2: $\mathrm{T}(65)=4.84, \mathrm{P}<0.001$; Exp. 7.2: $\mathrm{T}(70)=7.77, \mathrm{P}<0.001$;Exp. 8.2: $\mathrm{T}(72)=4.61$, $\mathrm{P}<0.001)$, however they are most of the time not significantly different from SP slopes (Exp. 5: $\mathrm{T}(70)=1.75, \mathrm{P}=1$; Exp. 6.2: $\mathrm{T}(65)=1.12, \mathrm{P}=1$; Exp. 7.2: $\mathrm{T}(70)=3.3, \mathrm{P}<0.05$;Exp. 8.2: $\mathrm{T}(72)=1.12, \mathrm{P}=1)$. SP slopes for their part, are systematically higher than $\mathrm{ES}$ slopes (Exp. 5: $\mathrm{T}(70)=4.05, \mathrm{P}<0.01$; Exp. 6.2: $\mathrm{T}(65)=5.34, \mathrm{P}<0.001$; Exp. 7.2: $\mathrm{T}(70)=4.8$, $\mathrm{P}<0.001$;Exp. 8.2: $\mathrm{T}(72)=4.62, \mathrm{P}<0.001)$, designating the $\mathrm{ES}$ values as the lowest slopes and the closest to 0 .
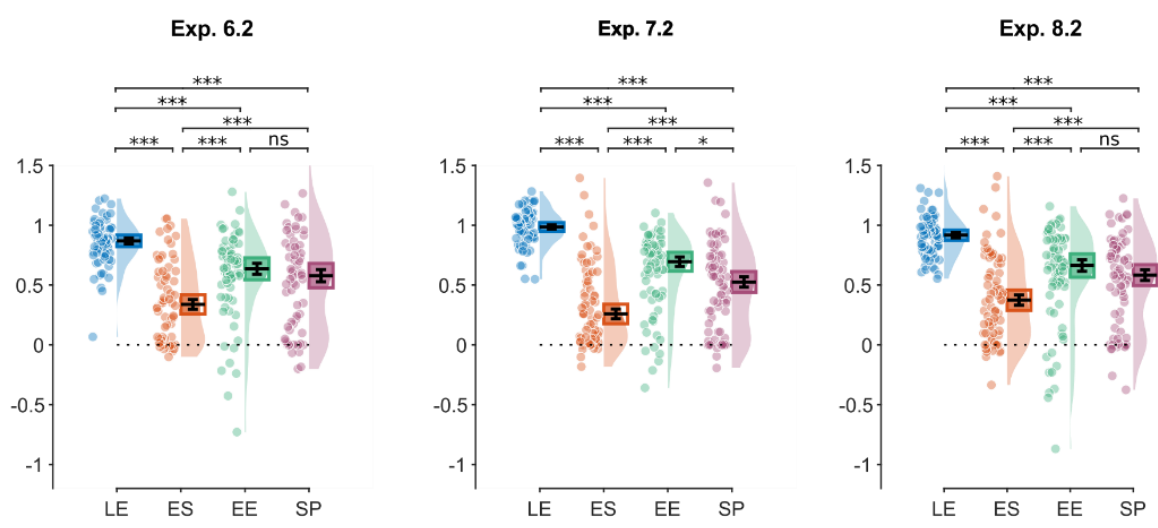

Learning (LE) $\quad \square$ Experiential-Symbolic (ES)

Experiential-Experiential (EE)

Stated Probability (SP) 
Fig S4. Inferred option values in Experiments 5-to-8. Comparison of individual inferred slopes obtained from linear fit in the 4 modalities (LE, ES, EE and SP in blue, orange, green and purple, respectively). The black lines represent mean and standard error of the mean. The colored boxes represent $95 \%$ confidence interval. The shaded area represents the probability density function. ${ }^{* * *} p<0.001$ paired sample t-tests.

\section{Choice profiling among Experiment 1-to-8}

We classified ES-choices in different categories as a function of being explained exclusively either by a full E-value neglect, by E-option estimates elicited in the LE phase, by both, or finally by none of them (Fig. S5).

In order to do so, we ran two simulations for each experiment.

In the first simulation, for each subject, we simulate an agent that is confronted with the history of decision problems that the real subject was facing. This artificial agent makes decisions according to the following experiential neglect decision rule: If the S-option is above $50 \%$ chance of winning a point, choose the S-option, otherwise choose the Eoption. This behavior is what we name experiential neglect, because the values of the $\mathrm{E}$ options are not even considered by the decision-maker.

In the second simulation, we also simulate an agent that is confronted with the history of decision problems that the real subject was facing. However, this agent has access to the E-option value estimates (specific to the subject in question) that were inferred from the LE-phase through our Q-learning model fitting procedure (see methods). We do not assume any deformation regarding the perception of S-option probabilities and rewards. Consequently, we assume that the agent uses an argmax rule (i.e., systematically choosing the highest value), to decide between the (subjective) E-option estimates and the S-option objective expected-value.

With this simulated choice dataset, we can compute the proportion of choices from our behavioral data that match with each simulation.

We observe that most of the choices can be both explained by LE estimates and the extreme experiential neglect decision rule (see main text). Yet, the number of choices 
1145 exclusively explained (or predicted) by the experiential neglect rule is significantly higher 1146 than the number of choices explained by LE estimates $(T(598)=13.87, P<0.001)$.

1147 Of note, the Exp. 7, due to its particular configuration of probabilities among E- and S1148 options, seemingly allows to discriminate better between the two decision models, as the 1149 number of choices explained by both decision rules.

Choices explained (\%)

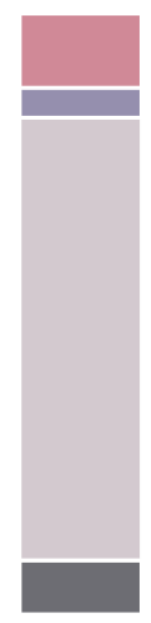

1

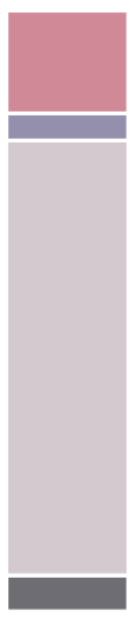

2

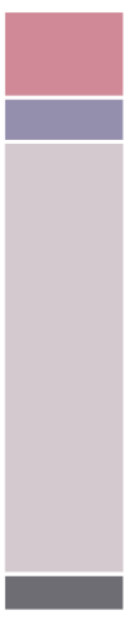

3

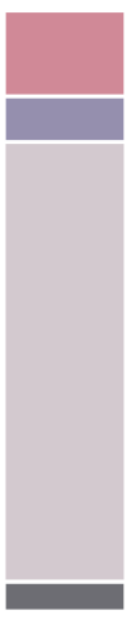

4

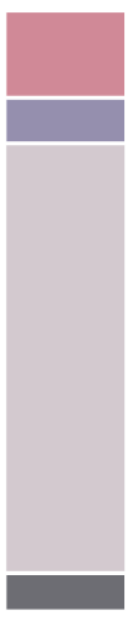

5

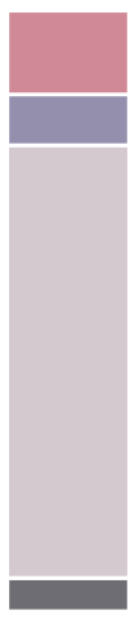

6

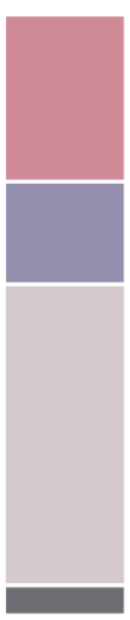

7

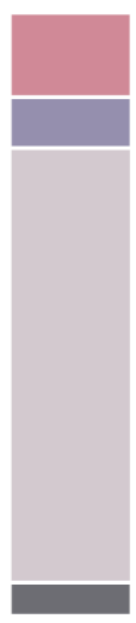

8

Exp.

1151
Fig 55. Choices prediction from Experiments 1-to-8. We run 2 simulations. In the first one, we assume that all participants make use of an experiential neglect decision rule, which basically consists in choosing the S-option as long as it is higher than $50 \%$ chance of winning a point, and otherwise choose the E-option. The second one consists in simulating all choices while assuming that participants use experiential values from the LE phase (i.e., the ones we inferred through our Q-learning model), therefore "LE estimates". Thereafter we compute the proportion of choices that are explained by each simulation, i.e., the proportion of behavioral choice that are identical to simulated choices. Choices explained by experiential neglect are in red. Choices explained by inferred experiential values from the LE phase are in dark blue. Choices explained by both experiential values and experiential neglect are in grey. Choices explained by none of them are in black. 


\section{References}

1164

1165

1166

1167

1168

1169

1170

1171

1172

1173

1174 1175

1176 1177

1178
1. W. M. DuCharme, M. L. Donnell, Intrasubject comparison of four response modes for "subjective probability" assessment. Organ. Behav. Hum. Perform. 10, 108-117 (1973).

2. R. D. Luce, The choice axiom after twenty years. J. Math. Psychol. 15, 215-233 (1977).

3. R. D. Luce, Individual choice behavior: A theoretical analysis (Courier Corporation, 2012).

4. T. E. J. Behrens, M. W. Woolrich, M. E. Walton, M. F. S. Rushworth, Learning the value of information in an uncertain world. Nat. Neurosci. 10, 1214-1221 (2007).

5. C. Findling, N. Chopin, E. Koechlin, Imprecise neural computations as a source of adaptive behaviour in volatile environments. Nat. Hum. Behav. 5, 99-112 (2021).

6. M. Lebreton, K. Bacily, S. Palminteri, J. B. Engelmann, Contextual influence on confidence judgments in human reinforcement learning. PLOS Comput. Biol. 15, e1006973 (2019).

7. R. C. Wilson, A. G. Collins, Ten simple rules for the computational modeling of behavioral data. elife. 8, e49547 (2019).

8. D. Frisch, J. Baron, Ambiguity and rationality. J. Behav. Decis. Mak. 1, 149-157 (1988). 